\title{
COMPLETELY UNSTABLE DYNAMICAL SYSTEMS
}

\author{
BY
}

\author{
SUDHIR K. GOEL AND DEAN A. NEUMANN
}

\begin{abstract}
We associate with the $C^{r}(r \geqslant 1)$ dynamical system $\phi$ on an $m$-manifold $M$, the orbit space $M / \phi$, defined to be the set of orbits of $\phi$ with the quotient topology. If $\phi$ is completely unstable, $M / \phi$ turns out to be a $C^{r}(m-1)$-nonseparated manifold. It is known that for a completely unstable flow $\phi$ on a contractible manifold $M, M / \phi$ is Hausdorff if and only if $\phi$ is parallelizable. In general, we place an order on the non-Hausdorff points of $M / \phi$ (essentially) by setting $\bar{p}<\bar{q}$ if and only if $\pi^{-1}(\bar{q}) \subseteq J^{+}\left(\pi^{-1}(\bar{p})\right)$. Our result is that $(M, \phi)$ is topologically equivalent to $\left(M^{\prime}, \phi^{\prime}\right)$ if and only if $M / \phi$ is order isomorphic to $M^{\prime} / \phi^{\prime}$.
\end{abstract}

0. Introduction. A dynamical system $\phi$ on an $m$-manifold $M$ is said to be completely unstable if it has no nonwandering points. Such systems occur very naturally. For example, on $\mathbf{R}^{2}$ any dynamical system without equilibria is completely unstable; and, of course, the restriction of an arbitrary dynamical system to the complement of its nonwandering set is completely unstable. All open manifolds admit completely unstable systems. The absence of nonwandering points does not imply the absence of recurrence phenomena, and it is well known that such systems can be extremely complex (cf. [TW, Ni1-Ni4 and HR]). There is an example due to Wazewski [Wa] of a vector field without singularities on $\mathbf{R}^{2}$ ! with every orbit of the corresponding dynamical system a separatrix.

Our aim is to classify completely unstable systems $(M, \phi)$ up to topological equivalence in terms of the associated orbit space $M / \phi$. Topologically $M / \phi$ is, as usual, the set of orbits of $\phi$ with the finest topology making the natural projection $\pi$ : $M \rightarrow M / \phi$ continuous. An additional order structure on $M / \phi$ is necessary if $M / \phi$ is to completely determine $(M, \phi)$. This order structure is required to specify the sequence in which the orbits of a prolongational limit set $J^{+}(x)$ are met by an orbit of $\phi$, and is defined essentially as follows: $\bar{p}<\bar{q}$ in $M / \phi$ if and only if $\pi^{-1}(\bar{q}) \subseteq$ $J^{+}\left(\pi^{-1}(\bar{p})\right)$ in $M$. (The actual order is necessarily more complex; the preceding description conveys the basic idea and enables us to state our main result without elaborate preliminaries.) The resulting ordered orbit space characterizes $(M, \phi)$.

Classification theorem. For $r \geqslant 1$, two $C^{r}$ completely unstable dynamical systems $(M, \phi)$ and $\left(M^{\prime}, \phi^{\prime}\right)$ are topologically equivalent if and only if the associated orbit spaces $M / \phi$ and $M^{\prime} / \phi^{\prime}$ are homeomorphic by an order preserving homeomorphism.

Received by the editors March 15, 1984 and, in revised form, January 13, 1985.

1980 Mathematics Subject Classification. Primary 58F18; Secondary 58F25.

(C1985 American Mathematical Society $0002-9947 / 85 \$ 1.00+\$ .25$ per page 
Essentially the same result, in the case where both $M, M^{\prime}=\mathbf{R}^{2}$, is given in Haefliger and Reeb [HR]; the result in the case when both $M$ and $M^{\prime}$ are 2-manifolds is given in [Ne1].

Under the assumption that $\phi$ is $C^{r}(r \geqslant 1)$ and completely unstable, the topology of $M / \phi$ is locally ( $m-1)$-Euclidean and has a countable basis; we refer to such a space as a nonseparated manifold. $M / \phi$ is a Hausdorff space (and hence a manifold in the usual sense) precisely in the case $\phi$ is parallelizable [Ma]. The classification theorem also holds (with the same proof) for $C^{0}$ systems, provided $m=\operatorname{dim}(M) \leqslant 3$. In case $m \geqslant 4$, there are pathological examples of completely unstable $C^{0}$ systems that do not admit local cross-sections that are locally Euclidean [Ne2], so that $M / \phi$ is not a nonseparated manifold. Our proof of the classification theorem does extend to $C^{0}$ systems that do admit locally Euclidean cross-sections, in all dimensions, but not to these pathological cases.

In $\S 1$, we give most of the definitions and notation required in the proof of the classification theorem; the proof itself occupies $\S \S 2-7$. Finally in $\S 8$, we give an application: we prove that the so-called Coleman conjecture (whether or not a flow on $\mathbf{R}^{m} \times \mathbf{R}^{n}$ with a topologically hyperbolic equilibrium is necessarily locally topologically equivalent to a flow with a differentiably hyperbolic (generic) rest point) is true in the case $m=1$ or $n=1$. The conjecture is known to be false in all other cases [Ne3, Pi].

\section{Preliminaries.}

1.1. Definitions and Notation. Throughout what follows $M$ denotes a $C^{r}$ $m$-manifold $r=0,1, \ldots, \infty ; \phi: M \times \mathbf{R}^{1} \rightarrow M$ denotes a $C^{r}$ dynamical system (flow) on $M$. We will use the following notation: $S \cdot T=\{\phi(x, t) \mid x \in S, t \in T\}$ for $S \subseteq$ $M$ and $T \subseteq \mathbf{R}^{1} ; x \cdot T=\{x\} \cdot T$; and $x \cdot t=\phi_{t}(x)=\phi(x, t)$ for $x \in M$ and $t \in \mathbf{R}^{1}$. The orbit of $x \in M$ is the set $\gamma(x)=x \cdot \mathbf{R}^{1} ; \gamma(x)$ inherits an orientation from the natural orientation of $\mathbf{R}^{1}$. Also throughout what follows, for any set $A \subseteq M$, Int $A=\AA$ and $\bar{A}$ will denote the interior and closure of $A$ in $M$ respectively.

If $\psi: N \times \mathbf{R}^{1} \rightarrow N$ is another dynamical system, we say that $\phi$ and $\psi$ are topologically equivalent if there is a homeomorphism $h$ of $M$ onto $N$ taking orbits of $\phi$ onto orbits of $\psi$ preserving orientation.

A set $U \subseteq M$ is said to be wandering (with respect to $\phi$ ) if there exists $t_{0} \in \mathbf{R}^{1}$ such that $U \cdot t \cap U=\varnothing$ for each $t$ with $|t| \geqslant t_{0}$. A point $x \in M$ is nonwandering if it has no wandering neighborhood. Equivalently, $x \in M$ is nonwandering if $x \in J^{+}(x)$, where $J^{+}(x)$ denotes the set of limits of sequences $\left\{x_{n} \cdot t_{n}\right\}$, where $\left\{x_{n}\right\}$ converges to $x$ and $\left\{t_{n}\right\}$ tends to $\infty$. For $A \subseteq M$ set $J^{+}(A)=\cup_{x \in A} J^{+}(x)$. The (closed $\phi$-invariant) set of all nonwandering points of $\phi$ will be denoted by $\Omega(\phi)$.

A flow $\phi$ is completely unstable if $\Omega(\phi)=\varnothing$. If $\phi$ is a completely unstable $C^{r}$ flow on an $m$-manifold $M$, a cross-section of $\phi$ is a set $S \subseteq M$ for which the mapping $h$ : $S \times \mathbf{R}^{1} \rightarrow M$ defined by $h(s, t)=s \cdot t$ is a homeomorphism of $S \times \mathbf{R}^{1}$ onto a subset of $M$. It is known that if $r \geqslant 1$, or if $r=0$ and $m \leqslant 3$ [Wh1, Wh2], then for any point $x \in M$ there is a cross-section $S$ satisfying the following conditions:

(a) $S$ is homeomorphic to the (closed) unit disk $D^{m-1} \subseteq \mathbf{R}^{m-1}$, and

(b) $x$ is in the relative interior of $S$. 
In the sequel we will always use the term "cross-section through $x$ " in the restricted sense of a cross-section satisfying (a) and (b).

A topological space is a nonseparated manifold if it is locally Euclidean and has a countable basis; the usual Hausdorff separation axiom is not assumed.

Given a dynamical system $M / \phi$ the orbit space $M / \phi$ is the set of orbits of $\phi$ with the quotient topology (the finest topology in which the natural projection: $\pi$ : $M \rightarrow M / \phi$ is continuous). For a completely unstable flow $\phi, M / \phi$ is well behaved, as is shown by the following result, which is essentially Theorem 3 of [Ma].

1.2. Proposition. If $\phi$ is a completely unstable $C^{r}(r \geqslant 1)$ flow on the m-manifold $M$, then $M / \phi$ is a nonseparated $(m-1)$-manifold and admits a unique $C^{r}$ differentiable structure with respect to which $\pi: M \rightarrow M / \phi$ is the projection of a locally trivial $C^{r}$ fiber bundle with fiber $\mathbf{R}^{\mathbf{1}}$.

1.3. Remarks. (a) In general $M / \phi$ will fail to be Hausdorff even when $\phi$ is completely unstable. In fact it is known [Ma, Proof of Theorem 4] that for a completely unstable flow $\phi$ on a contractible manifold $M, M / \phi$ is Hausdorff if and only if $\phi$ is parallelizable, i.e., if and only if there is a set $S \subseteq M$ (global crosssection) such that the mapping $h: S \times \mathbf{R}^{1} \rightarrow M$ defined by $h(x, t)=x \cdot t$ is a homeomorphism of $S \times \mathbf{R}^{1}$ onto $M$.

(b) Proposition 1.2 does not hold in general for $C^{0}$ flows. It does hold for $C^{0}$ flows if $m \leqslant 3$ [CO] but in all higher dimensions there are examples of $C^{0}$ completely unstable flows (in fact parallelizable flows) for which $M / \phi$ is not locally Euclidean. The reason is that local cross-sections of $C^{0}$ flows do not have to be locally Euclidean; examples are given in [Ch and Ne2] and are based on the original construction of Bing [Bi] of a nonmanifold $\bar{X}$ with the property that $X \times \mathbf{R}^{1}$ is homeomorphic to $\mathbf{R}^{4}$.

1.4. EXAmple. We given an example to motivate the introduction of an order structure on the orbit space. Consider the flows $\phi, \phi^{\prime}$ defined on $\mathbf{R}^{2}$ by the solutions of the following system of differential equations:

$(\phi)$ :

$$
\begin{gathered}
\dot{x}= \begin{cases}\sin ^{2} x, & 0 \leqslant x \leqslant 2 \pi, \\
0 & \text { elsewhere },\end{cases} \\
\dot{y}= \begin{cases}\cos x, & 0 \leqslant x \leqslant 2 \pi, \\
1 & \text { elsewhere }\end{cases} \\
\dot{x}= \begin{cases}\sin ^{2} x, & 0 \leqslant x \leqslant \pi, \\
-\sin ^{2} x, & \pi \leqslant x \leqslant 2 \pi, \\
0 & \text { elsewhere, }\end{cases} \\
\dot{y}= \begin{cases}\cos x, & 0 \leqslant x \leqslant 2 \pi, \\
1 & \text { elsewhere }\end{cases}
\end{gathered}
$$

The phase portraits of $\phi$ and $\phi^{\prime}$ are shown in Figure 1. We may determine the topological type of the orbit space $\mathbf{R}^{2} / \phi$ by considering the cross-sections indicated by the dotted lines in Figure 1(a). 


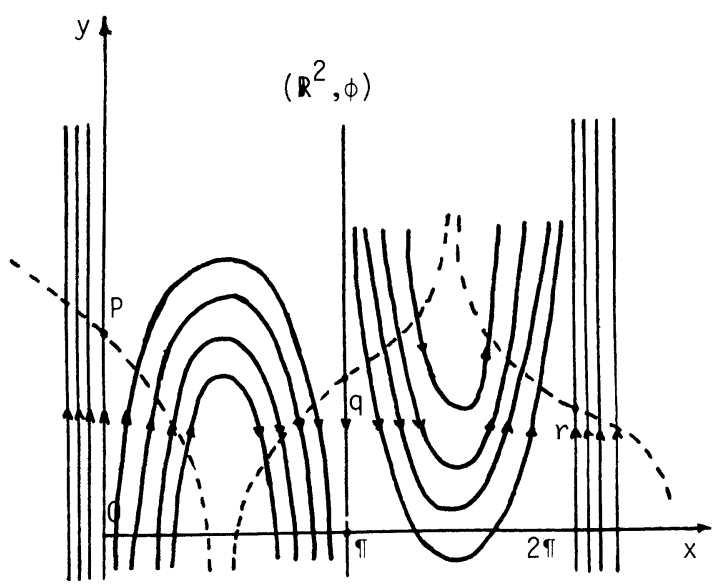

(a)
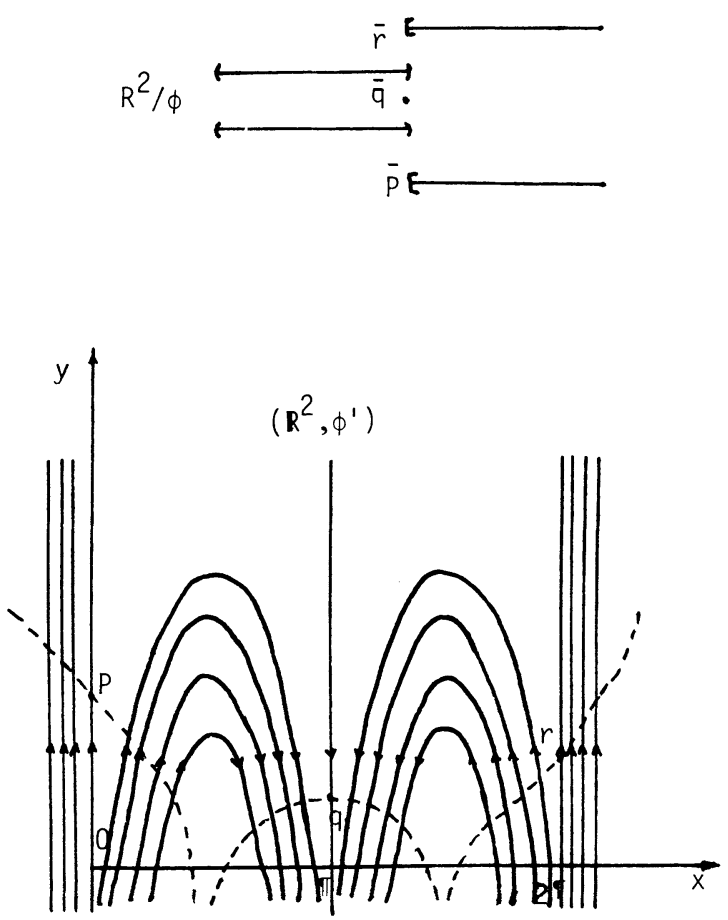

(b)

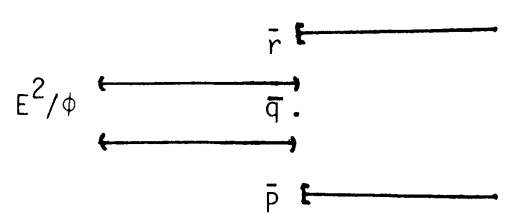

Figure 1 
It is clear that $\mathbf{R}^{2} / \phi$ is also the quotient of these three copies of $\mathbf{R}^{1}$, in which those pairs of points lying on the same orbit of $\phi$ are identified. The resulting nonseparated 1-manifold $\mathbf{R}^{2} / \phi$ is indicated schematically in the figure. Note that, while each point of $\mathbf{R}^{2} / \phi$ has neighborhoods homeomorphic to an open interval, neither of the pairs $\{\bar{p}, \bar{q}\},\{\bar{q}, \bar{r}\}$ can be separated with disjoint open sets.

These "non-Hausdorff" points $\bar{p}, \bar{q}, \bar{r}$ of $\mathbf{R}^{2} / \phi$ correspond to orbits that are separatrices in the terminology of [Ma]. $\mathbf{R}^{2} / \phi^{\prime}$ may be determined analogously.

Our aim is to classify completely unstable dynamical systems $(M, \phi)$ up to topological equivalence in terms of the orbit spaces $M / \phi$. Note that in the present example, $\left(\mathbf{R}^{2}, \phi\right)$ and $\left(\mathbf{R}^{2}, \phi^{\prime}\right)$ are not topologically equivalent; nevertheless $\mathbf{R}^{2} / \phi$ and $\mathbf{R}^{2} / \phi^{\prime}$ are homeomorphic. Thus we need to impose some additional structure on $M / \phi$ in order that the resulting space completely determines $(M, \phi)$ in the general case. In this example we can accomplish this by imposing a natural order relation on the non-Hausdorff points of the orbit space as follows:

$$
\bar{x}<\bar{y} \text { if and only if } \pi^{-1}(\bar{y}) \subseteq J^{+}\left(\pi^{-1}(\bar{x})\right) .
$$

The order imposed on $\mathbf{R}^{2} / \phi$ is then $\bar{p}<\bar{q}$ and $\bar{q}<\bar{r}$, while that imposed on $\mathbf{R}^{2} / \phi^{\prime}$ is $\bar{p}<\bar{q}$ and $\bar{r}<\bar{q}$. It is easy to verify that there is no order preserving homeomorphism between $\mathbf{R}^{2} / \phi$ and $\mathbf{R}^{2} / \phi^{\prime}$, so the ordered spaces are adequate to distinguish the flows $\phi$ and $\phi^{\prime}$. One can prove in fact, that two completely unstable flows on $\mathbf{R}^{2}$ are topologically equivalent if and only if there is an order preserving homeomorphism between the corresponding orbit spaces, ordered by $(*)$.

It turns out, however, that the natural order defined by $(*)$ is not sufficient for more general completely unstable flows. The modification that is required is the subject of the next section.

\section{The ordered orbit space $M / \phi$.}

2.1. Definition. Let $(M, \phi)$ be a completely unstable dynamical system with orbit space $M / \phi$. Let $\pi: M \rightarrow M / \phi$ denote the natural projection and for each $p \in M$, set $\bar{p}=\pi(p) \in M / \phi$. We define an order relation < on certain points of $M / \phi$ and on certain subsets of $M / \phi$ as follows:

(a) If $\bar{p}$ and $\bar{q}$ are distinct points of $M / \phi$, we say $\bar{p}<\bar{q}$ if and only if $\pi^{-1}(\bar{q}) \subseteq$ $J^{+}\left(\pi^{-1}(\bar{p})\right)$.

(b) Suppose $\bar{p}$ and $\bar{q}$ in $M / \phi$ satisfy $\bar{p}<\bar{q}$ and $\bar{q}<\bar{p}$. Given $A, B \subseteq M / \phi$ with $\bar{p} \in A, \bar{q} \in B$ and satisfying

(i) $A \backslash\{\bar{p}\}=B \backslash\{\bar{q}\}$;

(ii) $\bar{p} \in \overline{A \backslash\{\bar{p}\}}, \bar{q} \in \overline{B \backslash\{\bar{q}\}}$; and

(iii) $\bar{p}$ and $\bar{q}$ cannot be separated with disjoint open sets in $A \cup B$,

we say $A<B$ if and only if there are sections $S$ and $T$ lying over $A$ and $B$ respectively such that orbits of $\phi$ meet $S$ before they meet $T$.

The additional order structure given by (b) removes the ambiguity inherent in the possibility that we may have both $\bar{p}<\bar{q}$ and $\bar{q}<\bar{p}$ under the order defined by $J^{+}$ alone. This is made precise in the following proposition.

2.2. Proposition. Let $A, B, \bar{p}, \bar{q}$ be as in the definition, with $A<B$; then we cannot also have $B<A$. 
Proof. Let $S$ and $T$ be sections over $A$ and $B$ respectively, realizing $A<B$. Set $p=\pi^{-1}(\bar{p}) \cap S$ and $q=\pi^{-1}(\bar{q}) \cap T$. Let $\left\{p_{n}\right\}$ be a sequence in $S$ converging to $p$ and let $q_{n} \in \gamma\left(p_{n}\right) \cap T\left(n \in \mathbf{Z}^{+}\right)$, so $\left\{q_{n}\right\}$ converges to $q$. Then there is a sequence $\left\{t_{n}\right\}$ defined by $p_{n} \cdot t_{n}=q_{n}$. Note that we must have $\left\{t_{n}\right\} \rightarrow \infty$. For $t_{n}$ is positive by our assumption on $S$ and $T$; also if $\left\{t_{n}\right\}$ had a bounded subsequence, we could conclude $p \in \gamma(q) \subseteq J^{+}(\gamma(p))=J^{+}(p)$, contrary to our assumption that $\phi$ is completely unstable.

Now suppose $S^{\prime}$ and $T^{\prime}$ are arbitrary sections over $A$ and $B$ respectively. For each $n \in \mathbf{Z}^{+}$, set

$$
p_{n}^{\prime}=\gamma\left(p_{n}\right) \cap S^{\prime}, \quad q_{n}^{\prime}=\gamma\left(q_{n}\right) \cap T^{\prime},
$$

and define $t_{n}^{\prime}$ by $q_{n}^{\prime}=p_{n}^{\prime} \cdot t_{n}^{\prime}$. Then since $\left\{p_{n}^{\prime}\right\}$ converges to $\gamma(p) \cap S^{\prime},\left\{q_{n}^{\prime}\right\}$ converges to $\gamma(q) \cap T^{\prime}$, and $\left\{t_{n}\right\} \rightarrow \infty$, it follows that $\left\{t_{n}^{\prime}\right\} \rightarrow \infty$. Thus, at least for sufficiently large $n$, orbits of $\phi$ meet $S^{\prime}$ before they meet $T^{\prime}$. This proves the proposition.

2.3. Remarks. (a) We note that it follows from the proof of 2.2 that if $A<B$ and $S^{\prime}$ and $T^{\prime}$ are any disjoint connected sections over $A$ and $B$ respectively, then no orbit of $\phi$ can meet $T^{\prime}$ before it meets $S^{\prime}$.

(b) The requirement (b) in the definition of the order is undesirable but necessary. Examples are given in [Ne1] of flows $\phi, \phi^{\prime}$ on $\mathbf{R}^{2} \backslash\{0\}$ which are not distinguished by their orbit spaces ordered by $J^{+}$alone.

3. Statement of the classification theorem. Our main result is a complete classification of $C^{r}$ completely unstable flows on open manifolds in terms of the associated ordered orbit space.

3.1. Classification THEOREM. If $\phi$ and $\phi^{\prime}$ are completely unstable $C^{r}$ flows $(r \geqslant 1)$ on $m$-manifolds $M$ and $M^{\prime}$ respectively, then $(M, \phi)$ and $\left(M^{\prime}, \phi^{\prime}\right)$ are topologically equivalent if and only if $M / \phi$ is order isomorphic to $M^{\prime}, \phi^{\prime}$.

3.2. REmarKs. (a) This result for $M=M^{\prime}=\mathbf{R}^{2}$ is stated by Haefliger and Reeb in [HR]. It is proved for $C^{0}$ flows on 2-manifolds in [Ne1].

(b) Theorem 3.1 also holds for $C^{0}$ flows (with the same proof) provided $m \leqslant 3$, or for arbitrary $m$, provided we assume that $\phi$ and $\phi^{\prime}$ admit cross-sections at every point that are locally Euclidean, so that $M / \phi$ and $M^{\prime}, \phi^{\prime}$ are nonseparated manifolds.

3.3. Discussion. The fact that the ordered orbit space is an invariant of the topological equivalence class of $(M, \phi)$ follows immediately from the definitions. The proof of the converse occupies $\S \S 4-7$. Since this proof is rather lengthy, we conclude this section with a brief sketch which the reader may find helpful. Our aim is to lift a given order preserving homeomorphism $h: M^{\prime} / \phi^{\prime} \rightarrow M / \phi$ to a topological equivalence $k:\left(M^{\prime}, \phi^{\prime}\right) \rightarrow(M, \phi)$. We note that both $\pi: M \rightarrow M / \phi$ and $\pi^{\prime}: M^{\prime} \rightarrow$ $M^{\prime} / \phi^{\prime}$ are projections of locally trivial $\mathbf{R}^{1}$ bundles with structure group the group $\mathscr{T}$ of all translations of $R^{1}$; in this setting we seek a bundle map $k$ [St, §2.5] covering $h$. We first construct explicit parametrizations of both bundles. For $(M, \phi)$ this 
consists of an atlas $\left\{T_{n} \mid n \in \mathbf{Z}^{+}\right\}$for $M / \phi$, cross-sections $\alpha_{n}: T_{n} \rightarrow M, S_{n}=\alpha_{n}\left(T_{n}\right)$ of $\phi$, and "time" maps $f_{i j}: T_{i} \cap T_{j} \rightarrow \mathbf{R}^{1}$ defined by

$$
\phi\left(\alpha_{i}(x), f_{i j}(x)\right)=\alpha_{j}(x) \quad\left(x \in T_{i} \cap T_{j} ; i, j \in \mathbf{Z}^{+}\right) .
$$

Then $\pi^{-1}\left(T_{i}\right)$ is parametrized by $\psi_{i}: T_{i} \times \mathbf{R}^{1} \rightarrow \pi^{-1}\left(T_{i}\right)$ :

$$
\psi_{i}(x, t)=\phi\left(\alpha_{i}(x), t\right) .
$$

The coordinate transformations are then given by $\psi_{j}^{-1} \circ \psi_{i}(x, t)=\left(x, t+f_{j i}(x)\right)$, so the structure of the bundle $\pi: M \rightarrow M / \phi$ is determined by the maps $g_{i j}: T_{i} \cap T_{j} \rightarrow \mathscr{T}$, where $g_{i j}$ assigns $x$ in $T_{i} \cap T_{j}$ to the translation by $f_{i j}(x)$ in $\mathscr{T}$.

The bundle $\left(M^{\prime}, \phi^{\prime}\right)$ is parametrized analogously by an atlas $\left\{T_{n}^{\prime} \mid n \in \mathbf{Z}^{+}\right\}$crosssections $S_{n}^{\prime}=\alpha_{n}^{\prime}\left(T_{n}^{\prime}\right)$, and maps $f_{i j}^{\prime}: T_{i}^{\prime} \cap T_{j}^{\prime} \rightarrow \mathbf{R}^{1}, g_{i j}^{\prime}: T_{i}^{\prime} \cap T_{j}^{\prime} \rightarrow \mathscr{T}$. We first arrange that the two parametrizations are compatible under $h$, i.e., $T_{n}^{\prime}=h^{-1}\left(T_{n}\right)$ $\left(n \in \mathbf{Z}^{+}\right)$and

$$
\operatorname{sgn} f_{i j}(x)=\operatorname{sgn} f_{i j}^{\prime}\left(h^{-1}(x)\right)
$$

for all values of $i, j, x$, where both sides are defined. (This simply means that each pair $S_{i}, S_{j}$ of cross-sections of $\phi$ is related by $\phi$ in the same way the corresponding cross-sections $S_{i}^{\prime}, S_{j}^{\prime}$ are related by $\phi^{\prime}$; viz., a $\phi$ orbit $\pi^{-1}(x)$ meets $S_{i}$ ahead of $S_{j}$ if and only if the corresponding $\phi^{\prime}$ orbit $\pi^{\prime-1}\left(h^{-1}(x)\right)$ meets $S_{i}^{\prime}$ ahead of $S_{j}^{\prime}$.) We next show that we can reparametrize the flow $\phi$ in this situation so that in fact $f_{i j}(x)=f_{i j}^{\prime}\left(h^{-1}(x)\right)$, again for all relevant values of $i, j, x$. The required bundle equivalence $k$ may then be defined locally by

$$
k\left(\psi_{i}^{\prime}\left(x^{\prime}, t\right)\right)=\psi_{i}\left(h\left(x^{\prime}\right), t\right) .
$$

It is easily checked that $k$ is a well-defined homeomorphism of $M^{\prime}$ onto $M$. Thus $\phi^{\prime}$ is topologically equivalent to the reparametrized version of $\phi$, and hence to $\phi$ itself.

4. Covering systems of cross-sections. In this section $\phi$ denotes a $C^{r}$ completely unstable flow on an $m$-manifold $M$, with $r \geqslant 1$. We construct a locally finite collection of cross-sections of $\phi$ whose images under the natural projection $\pi$ : $M \rightarrow M / \phi$ cover $M / \phi$.

4.1. Definition. Fix $\varepsilon>0$ and let $I_{\varepsilon}=(-\varepsilon, \varepsilon)$. A neighborhood $U_{p}$ of a point $p \in M$ is called an $\varepsilon$-flow box at $p$ if there is a cross-section $S \subseteq M$ with $p \in S$ and such that the mapping $h: S \times I_{\varepsilon} \rightarrow M$ defined by $h(s, t)=s \cdot t$ is a homeomorphism of $S \times I_{\varepsilon}$ onto $U_{p}$.

It is known [BS, Theorem 2.9] that every point $p \in M$ admits an $\varepsilon$-flow box for which $S$ is homeomorphic to the closed disk $D^{m-1}$ of dimension $m-1$.

4.2. Definition. A collection $\left\{S_{i} \mid i \in \mathbf{Z}^{+}\right\}$of cross-sections of $\phi$ is called a covering system for $(M, \phi)$ if it satisfies the following conditions:

(a) each $S_{i}$ is homeomorphic with $D^{m-1}$;

(b) $\left\{S_{i} \mid i \in \mathbf{Z}^{+}\right\}$is locally finite;

(c) $\bigcup\left\{\dot{S}_{i} \mid i \in \mathbf{Z}^{+}\right\} \cdot \mathbf{R}^{1}=M$; and

(d) the $S_{i}$ are pairwise disjoint.

Note that according to our definition of cross-section, no orbit of $\phi$ meets $S_{i}$ more than once. 
4.3. Lemma. Any $C^{r}$ completely unstable dynamical system $(M, \phi)$ with $r \geqslant 1$ admits a covering system of cross-sections.

Proof. Since $M$ is $\sigma$-compact, we may write $M=\bigcup\left\{\stackrel{\circ}{M}_{k} \mid k \in \mathbf{Z}^{+}\right\}$, where each $M_{k}$ is compact, $M_{k} \subseteq \stackrel{\circ}{M}_{k+1}$ and $M_{1}=\varnothing$. Let $R_{1} \subseteq T_{1}$ be cross-sections of $\phi$, both homeomorphic to $D^{m-1}$, and so that the corresponding flow boxes $G_{1}=R_{1} \cdot I_{\varepsilon}$ and $H_{1}=T_{1} \cdot I_{\varepsilon^{\prime}}$ satisfy $\bar{G}_{1} \subseteq \stackrel{\circ}{H}_{1}$.

We now construct inductively a locally finite cover of $M$ by flow box pairs analogous to $\left(G_{1}, H_{1}\right)$. Suppose that we have determined integers $1=k_{1}<k_{2}<$ $\cdots k_{n}$, cross-sections $R_{i} \subseteq T_{i}$ of $\phi\left(1 \leqslant i \leqslant k_{n}\right)$ each homeomorphic to $D^{m-1}$, and corresponding flow boxes $G_{i}=R_{i} \cdot I_{\varepsilon_{i}}, H_{i}=T_{i} \cdot I_{\varepsilon_{i}^{\prime}}$ satisfying the following:

(a) $\bar{G}_{i} \subseteq \stackrel{\circ}{H}_{i}$ for $1 \leqslant i \leqslant k_{n}$;

(b) for each $j \leqslant n-1, M_{j+1} \backslash \stackrel{\circ}{M}_{j} \subseteq \bigcup\left\{\stackrel{\circ}{G}_{i} \mid k_{j}<i \leqslant k_{j+1}\right\}$; and

(c) for each $2 \leqslant j \leqslant n-1, M_{j-1} \cap \bigcup\left\{\bar{H}_{i} \mid k_{j}<i \leqslant k_{j+1}\right\}=\varnothing$.

Now for each $x \in M_{n+1} \backslash M_{n}$ choose cross-sections $R_{x} \subseteq T_{x}$ through $x$, each homeomorphic to $D^{m-1}$, and flow boxes $G_{x}=R_{x} \cdot I_{\varepsilon_{x}}, H_{x}=T_{x} \cdot I_{\varepsilon_{x}^{\prime}}$, so that $x \in \dot{G}_{x}$ $\subseteq \bar{G}_{x} \subseteq \stackrel{\circ}{H}_{x}$ and $\overline{H_{x}} \cap M_{n-1}=\varnothing$. Finitely many of $\dot{G}_{x}$ cover $M_{n+1} \backslash \dot{M}_{n}$; replace the corresponding indices with integers $k_{n}+1, \ldots, k_{n+1}$. By induction, we obtain a collection $\left\{T_{i} \mid i \in \mathbf{Z}_{+}\right\}$of cross-sections satisfying (a)-(c) of 4.2.

Of course the $T_{i}$ need not be pairwise disjoint, so we now modify the collection $\left\{T_{i}\right\}$ to obtain a disjoint family. We again proceed by induction; thus suppose that we have constructed cross-sections $S_{1}, S_{2}, \ldots, S_{l(n)}$ satisfying:

(a) each $S_{i}$ is homeomorphic to $D^{m-1}$;

(b) each $S_{i}$ is contained in some $H_{j}, j \leqslant n$;

(c) $\bigcup\left\{S_{i} \mid i \leqslant l(n)\right\} \cdot \mathbf{R}^{1} \supseteq \bigcup\left\{R_{j} \mid j \leqslant n\right\} \cdot \mathbf{R}^{1}$; and

(d) the $S_{i}$ are pairwise disjoint.

Fix $p \in R_{n+1}$. Choose $q \in p \cdot I_{\varepsilon_{n+1}}$ so that $q \notin R_{n+1}$ and $q$ is in none of the $S_{i}$ $(i \leqslant l(n))$, say $q=p \cdot t_{p}\left(t_{p} \in I_{\varepsilon_{n+1}}\right)$. Choose a flow box $S_{p} \cdot I_{\varepsilon_{p}} \subseteq T_{n+1} \cdot I_{\varepsilon_{n+1}}$ so that $S_{p}$ is homeomorphic to $D^{m-1}, q \in S_{p}, S_{p} \cdot t \subseteq T_{n+1}$ for some $t \in I_{\varepsilon_{n+1}}$ and that $S_{p}$ is disjoint from all the $S_{i}(i \leqslant l(n))$ (Figure 2).

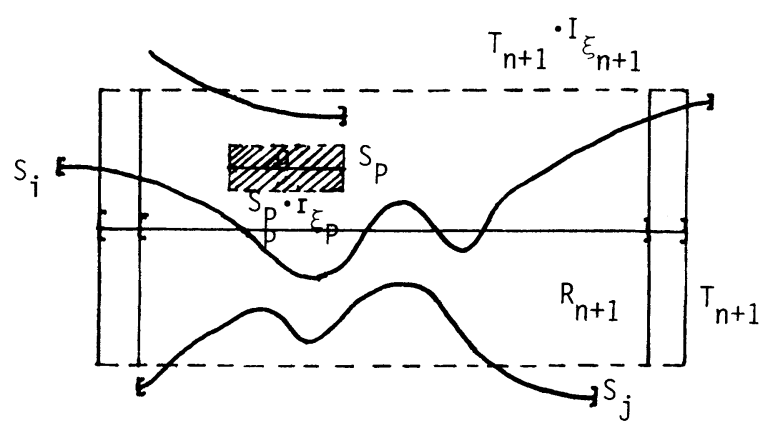

Figure 2 
Let $\pi: T_{n+1} \cdot \mathbf{R}^{1} \rightarrow T_{n+1}$ denote the natural projection. Since $R_{n+1}$ is compact, finitely many of the projections $\left\{\pi\left(S_{p}\right) \mid p \in R_{n+1}\right\}$ cover $R_{n+1}$. Label the corresponding cross-sections

$$
S_{l(n)+1}, S_{l(n)+2}, \ldots, S_{l(n+1)}
$$

Note that if the $S_{i}(l(n)+1 \leqslant i \leqslant l(n+1))$ are not already pairwise disjoint among themselves, they may be made so by small translations along the flow since they are all parallel to $T_{n+1}$, and that this may be done without introducing any intersections with the $S_{i}(i \leqslant l(n))$. By induction, we obtain the collection $\left\{S_{i} \mid i \in \mathbf{Z}^{+}\right\}$; it is easily checked that this collection satisfies (a)-(d) of 4.2 .

4.4. EXAmple. A natural question now arises as to the possibility of further simplifying a covering system of cross-sections. The proof of the classification theorem would be greatly simplified, for example, if one could always obtain a covering system $\left\{S_{i} \mid i \in \mathbf{Z}^{+}\right\}$whose family $\left\{\pi\left(S_{i}\right) \mid i \in \mathbf{Z}^{+}\right\}$of projections was a locally finite (or even point finite) cover of $M / \phi$. We give an example to show that this is not possible in general.

Consider the manifold $\hat{M}=I \times \mathbf{R}^{1}$, where $I=[0,1]$. Let $\left\{r_{n} \mid n \in \mathbf{Z}^{+}\right\}$be an enumeration of rationals in $I$ with $r_{1}=0$. Let $a_{n}=\left(r_{n}, n\right) \in \hat{M}$ for each $n \in \mathbf{Z}^{+}$. Let $M=\hat{M} \backslash \bigcup\left\{a_{n} \mid n \in \mathbf{Z}^{+}\right\}$. Let $\phi$ be a flow on $M$ defined (in Cartesian coordinates) by the differential equations

$$
\dot{x}=0, \quad \dot{y}=f(x, y), \quad x, y \in \hat{M},
$$

where $f: \hat{M} \rightarrow[0,1]$ is a smooth function that vanishes exactly on the set $\left\{a_{n} \mid n \in\right.$ $\left.\mathbf{Z}^{+}\right\}$. The phase portrait is as shown in Figure 3.

Let $\left\{S_{n} \mid n \geqslant 1\right\}$ be a covering system of cross-sections such that each $S_{i} \cong I$. We show that there exist orbits of $\phi$ which meet infinitely many cross-sections. Without loss of generality, let the cross-section $S_{1}$ be the compact interval $[0,1]$. For each $j$, let $p_{j}$ denote the projection on $S_{1}$ of the right-hand endpoint of the section $S_{j}$.

Since $\left\{S_{n}\right\}$ is a covering system, there exists a cross-section, say $S_{2}$, covering the orbit through $\left(r_{1}, 2\right)$ and extending to the right of it as well. Pick an irrational number $q_{2}$ in the open interval $\left(0, p_{2}\right)$. Note that the orbit through $\left(q_{2}, 0\right)$ meets at least two sections (Figure 4). Now pick a rational number, say $r_{k_{2}}$, in the interval $\left(0, q_{2}\right)$ such that $g_{12}\left(r_{k_{2}}\right)$ is finite, where $g_{i j}(x)$ denotes the time along the orbit of $x$ from $S_{i}$ to $S_{j}$. Thus the orbit through the point $\left(r_{k_{2}}, 0\right)$ meets at least two sections.

As above, there exists a section, say $S_{3}$, covering the orbit through $\left(r_{k_{2}}, k_{2}+1\right)$ and extending to the right of it as well. Pick an irrational number $q_{3}$ in the interval $\left(r_{k_{2}}, \min \left(q_{2}, p_{3}, r_{k_{2}}+\frac{1}{2}, 1\right)\right)$. Observe that the orbit through $\left(q_{3}, 0\right)$ meets at least three sections.

Continuing this process by induction, we obtain a nested sequence

$$
\left(0, q_{2}\right] \supsetneqq\left(r_{k_{2}}, q_{3}\right] \supsetneqq\left(r_{k_{3}}, q_{4}\right] \cdots
$$




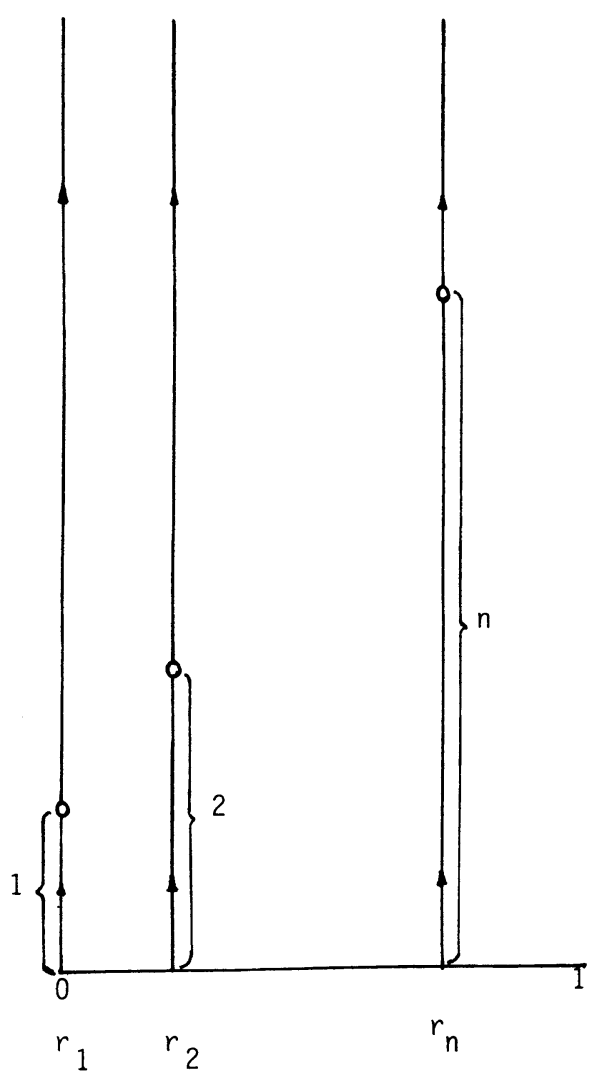

Figure 3

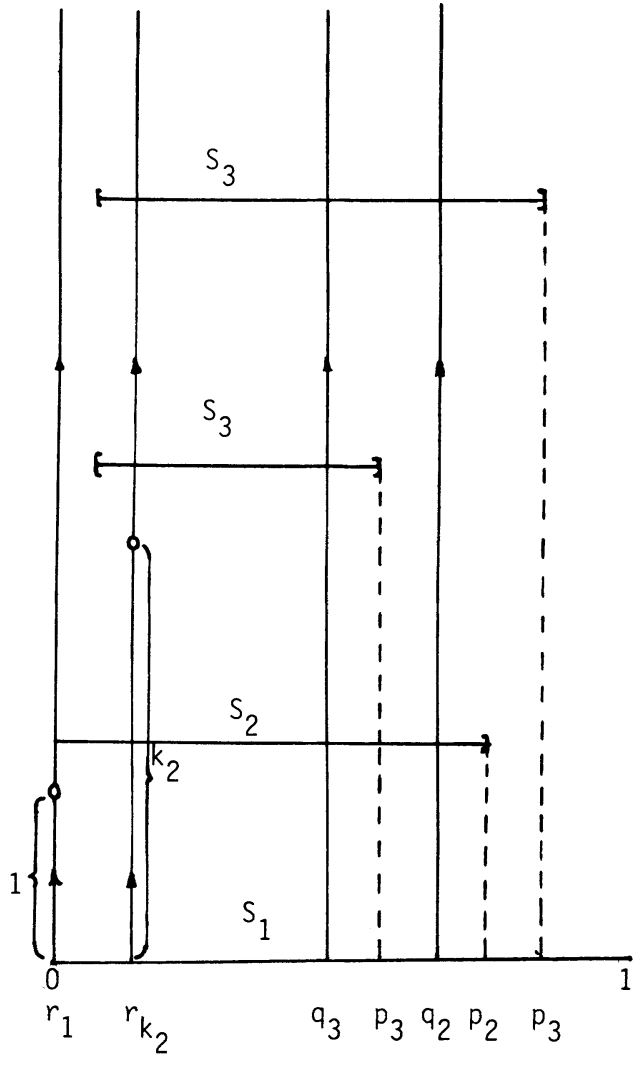

FIGURE 4

of intervals such that the orbits through both $\left(r_{k_{j}}, 0\right)$ and $\left(q_{j}, 0\right)$ meet at least $j$ sections. Moreover, from the above construction, we have

$$
\bigcap_{i \geqslant 1}\left(r_{k_{i}}, q_{i+1}\right]=\bigcap_{i \geqslant 1}\left[r_{k_{i}}, q_{i+1}\right] \quad\left(\text { where } r_{k_{1}}=0\right) .
$$

Since $[0,1]$ is a complete metric space and $r_{k_{i}}, q_{i}$ are so chosen that $\operatorname{diam}\left[r_{k_{i}}, q_{i+1}\right] \leqslant$ $1 / 2^{i-1}, \bigcap_{i \geqslant 1}\left[r_{k_{i}}, q_{i+1}\right]$ contains exactly one point, say $p$. If $p$ is an irrational point, the orbit of $(p, 0)$ meets infinitely many sections; if $p=\left(r_{k}, 0\right)$ for some $k$, then the orbit through $\left(r_{k}, k+1\right)$ meets infinitely many sections.

5. Compatible systems of cross-sections. We now consider two $C^{r}$ completely unstable dynamical systems $\left(M^{\prime}, \phi^{\prime}\right),(M, \phi)$ and a given order preserving homeomorphism $h: M^{\prime} / \phi^{\prime} \rightarrow M / \phi$ of the corresponding orbit spaces, ordered as in $\S 2$. The object of the present section is to obtain a covering system $\left\{S_{n}^{\prime} \mid n \in \mathbf{Z}^{+}\right\}$of cross-sections of $\phi^{\prime}$ and a covering system $\left\{S_{n} \mid n \in \mathbf{Z}^{+}\right\}$of cross-sections of $\phi$ that are compatible in the sense that the projections $\left\{\pi^{\prime} S_{n}^{\prime}\right\}$ and $\left\{\pi S_{n}\right\}$ correspond for each $n$ under $h$, and that for any pair $(i, j)$ of indices, $S_{i}$ and $S_{j}$ are related by $\phi$ in the same way that the corresponding sections $S_{i}^{\prime}$ and $S_{j}^{\prime}$ are related by $\phi^{\prime}$. We make this precise in the following definition. 
5.1. Definition. For each $n \in \mathbf{Z}^{+}$, set $T_{n}^{\prime}=\pi^{\prime}\left(S_{n}^{\prime}\right)$, where $\pi^{\prime}: M^{\prime} \rightarrow M^{\prime} / \phi^{\prime}$ is the natural projection; let $\alpha_{n}^{\prime}: T_{n}^{\prime} \rightarrow M^{\prime}$ be the (unique) continuous map with $\pi^{\prime} \circ \alpha_{n}^{\prime}=1$ and $\alpha_{n}^{\prime}\left(T_{n}^{\prime}\right)=S_{n}^{\prime}$. For each pair $(i, j)$ set $A_{i j}^{\prime}=T_{i}^{\prime} \cap T_{j}^{\prime}$ and define $f_{i j}^{\prime}: A_{i j}^{\prime} \rightarrow \mathbf{R}^{1}$ by $\phi^{\prime}\left(\alpha_{i}^{\prime}\left(x^{\prime}\right), f_{i j}^{\prime}\left(x^{\prime}\right)\right)=\alpha_{j}^{\prime}\left(x^{\prime}\right)$; (so $f_{i j}^{\prime}(x)$ is the "time" along the orbit $\pi^{\prime-1}\left(x^{\prime}\right)$ from $S_{i}^{\prime}$ to $\left.S_{j}^{\prime}\right)$. We note that $f_{i j}^{\prime}$ is continuous on its domain.

For $n \in \mathbf{Z}^{+}$, set $T_{n}=h\left(T_{n}^{\prime}\right)$. Suppose $\left\{S_{n} \mid n \in \mathbf{Z}^{+}\right\}$is a covering system of cross-sections of $\phi$, with $\pi\left(S_{n}\right)=T_{n}$ for each $n \in \mathbf{Z}^{+}$. Define $\alpha_{n}, f_{i j}, A_{i j}$ as for $\left(M^{\prime}, \phi^{\prime}\right)$. We say that $\left\{S_{n}\right\}$ is compatible with $\left\{S_{n}^{\prime}\right\}$ if, for each pair $(i, j)$ of indices, we have

(c) $\operatorname{sgn} f_{i j}(\mathrm{x})=\operatorname{sgn} f_{i j}^{\prime}\left(h^{-1}(x)\right)\left(x \in A_{i j}\right)$.

5.2. Proposition. If $\left(M^{\prime}, \phi^{\prime}\right),(M, \phi)$ are $C^{r}(r \geqslant 1)$ completely unstable dynamical systems, and $h: M^{\prime} / \phi^{\prime} \rightarrow M / \phi$ is an order preserving homeomorphism of the corresponding orbit spaces, then there exist compatible covering systems of cross-sections $\left\{S_{n} \mid n \in \mathbf{Z}^{+}\right\}$of $(M, \phi)$ and $\left\{S_{n}^{\prime} \mid n \in \mathbf{Z}^{+}\right\}$of $\left(M^{\prime}, \phi^{\prime}\right)$.

The proof of this proposition is somewhat lengthy so we break it up into several sections. We begin with two preliminary results, a general lemma (5.4) on separation of semicontinuous functions by continuous functions, and a technical lemma (5.5) that isolates the essential consequence of the fact that $h$ preserves order. We next fix a covering system $\left\{S_{n}^{\prime} \mid n \in \mathbf{Z}^{+}\right\}$for $\left(M^{\prime}, \phi^{\prime}\right)$ and construct liftings $\alpha_{n}: T_{n} \rightarrow M$ $\left(T_{n}=h\left(T_{n}^{\prime}\right), T_{n}^{\prime}=\pi\left(S_{n}^{\prime}\right)\right)$ and corresponding cross-sections $S_{n}=\alpha_{n}\left(T_{n}\right)$ of $(M, \phi)$ that satisfy (c), but neglecting temporarily the requirement of local finiteness (5.6-5.8). Finally we modify the system of cross-sections to achieve local finiteness without disturbing (c) (5.9-5.11). At this stage it is necessary to modify also the system $\left\{S_{n}^{\prime}\right\}$; this is the reason 5.2 does not assert that any covering system $\left\{S_{n}^{\prime}\right\}$ corresponds to a compatible system $\left\{S_{n}\right\}$.

5.3. Notation. Throughout this section $(M, \phi)$ and $\left(M^{\prime}, \phi^{\prime}\right)$ denote $C^{r}$ completely unstable dynamical systems, and $h: M^{\prime} / \phi^{\prime} \rightarrow M / \phi$ is an order preserving homeomorphism. Since we will often need to refer to points or subsets of $M / \phi$ and $M^{\prime} / \phi^{\prime}$ that correspond under $h$, it will be convenient to adopt the notation:

$$
x^{\prime}=h^{-1}(x) \quad(x \in M / \phi) ; \quad T^{\prime}=h^{-1}(T) \quad(T \subseteq M / \phi) .
$$

5.4. Separation lemma (C. H. Dowker). Let $Y$ be paracompact. Assume that $f$ is a lower, and $g$ is an upper semicontinuous real-valued function on $Y$ such that $g(y)<f(y)$ for each $y \in Y$. Then there exists a continuous function $h: Y \rightarrow \mathbf{R}^{1}$ such that $g(y)<$ $h(y)<f(y)$ for each $y \in Y$.

For a brief proof of this theorem, see [Du, §4.3, Chapter 8].

5.5. LEMMA. Let $f_{12}: T_{1} \cap T_{2} \rightarrow \mathbf{R}^{1}$ be the time map defined by arbitrary (compact) sections $S_{1}, S_{2}$ of $(M, \phi) \quad\left(\right.$ so $T_{i} \subseteq M / \phi, \alpha_{i}: T_{i} \rightarrow M, S_{i}=\alpha_{i}\left(T_{i}\right) \quad(i=1,2)$, $\phi\left(\alpha_{1}(x), f_{12}(x)\right)=\alpha_{2}(x)$ as above $)$. Let $f_{12}^{\prime}$ be the time map defined by arbitrary sections $S_{1}^{\prime}, S_{2}^{\prime}$ of $\left(M^{\prime}, \phi^{\prime}\right)$ over the corresponding sets $T_{1}^{\prime}, T_{2}^{\prime}$ in $M^{\prime} / \phi^{\prime}$. Suppose there is a sequence $\left\{x_{k}\right\} \subseteq T_{1} \cap T_{2}$ with $\left\{x_{k}\right\} \rightarrow x_{0} \in T_{1}$ and $\lim _{k \rightarrow \infty} f_{12}\left(x_{k}\right)=+\infty$ $(-\infty)$. Then $\lim _{k \rightarrow \infty} f_{12}^{\prime}\left(x_{k}^{\prime}\right)=+\infty(-\infty$ respectively $)$. 
Proof. We consider the case $\lim _{k \rightarrow \infty} f_{12}\left(x_{k}\right)=+\infty$, the other case being similar. We first assert that no limit point of the sequence $\left\{\alpha_{2}\left(x_{k}\right)\right\}$ can be in $\pi^{-1}\left(x_{0}\right)$. For if $q$ is such a limit point, then $q \in J^{+}(p)$ where $p=\alpha_{1}\left(x_{0}\right)=\pi^{-1}\left(x_{0}\right) \cap S_{1}$, so if also $q \in \pi^{-1}\left(x_{0}\right)=\gamma(p)$, then $p \in J^{+}(p)$ contrary to our assumption that $\phi$ is completely unstable. Note that this implies $x_{\mathrm{o}} \notin T_{2}$.

We can now verify that $\left\{f_{12}^{\prime}\left(x_{k}^{\prime}\right)\right\}$ has no bounded subsequence. For suppose that $f_{12}^{\prime}\left(x_{j}^{\prime}\right) \rightarrow t^{\prime} \in \mathbf{R}^{1}$; then

$$
\alpha_{2}^{\prime}\left(x_{j}^{\prime}\right)=\phi^{\prime}\left(\alpha_{1}^{\prime}\left(x_{j}^{\prime}\right), f_{12}^{\prime}\left(x_{j}^{\prime}\right)\right) \rightarrow \phi^{\prime}\left(\alpha_{1}^{\prime}\left(x_{0}^{\prime}\right), t^{\prime}\right) \in S_{2}^{\prime},
$$

and this implies that $x_{0}^{\prime} \in T_{2}^{\prime}$, hence $x_{0} \in T_{2}$, contrary to the result of the preceding paragraph.

Thus the only possibility contrary to the desired conclusion is that $\left\{f_{12}^{\prime}\left(x_{k}^{\prime}\right)\right\}$ has a subsequence that tends to $-\infty$. Using the compactness of $S_{2}, S_{2}^{\prime}$ and taking further subsequences, we can then obtain a sequence $\left\{x_{j}\right\} \subseteq T_{1} \cap T_{2}$ with the following properties:

(i) $\left\{x_{j}\right\} \rightarrow x_{0}$ and $f_{12}\left(x_{j}\right) \rightarrow+\infty$;

(ii) $\left\{x_{j}^{\prime}\right\} \rightarrow x_{0}^{\prime}$ and $f_{12}^{\prime}\left(x_{j}^{\prime}\right) \rightarrow-\infty$;

(iii) $\alpha_{2}\left(x_{j}\right) \rightarrow q \in S_{2}$; and

(iv) $\alpha_{2}^{\prime}\left(x_{j}^{\prime}\right) \rightarrow q^{\prime} \in S_{2}^{\prime}$.

Set $y_{0}=\pi(q), y_{0}^{\prime}=\pi^{\prime}\left(q^{\prime}\right)$. Since $\left\{x_{j}\right\}$ converges to $y_{0}$ in $T_{2}$ and $x_{j}^{\prime}$ converges to $y_{0}^{\prime}$ in $T_{2}^{\prime}$, we have $h\left(y_{0}^{\prime}\right)=y_{0}$. Now (i) and (iii) imply $x_{0}<y_{0}$; (ii) and (iv) imply $y_{0}^{\prime}<x_{0}^{\prime}$ and since $h$ preserves order this implies $y_{0}<x_{0}$. Now choose $j_{0}$ so that $f_{12}\left(x_{j}\right)>0\left(\right.$ all $\left.j \geqslant j_{0}\right)$ and $f_{12}^{\prime}\left(x_{j}^{\prime}\right)<0\left(\right.$ all $\left.j \geqslant j_{0}\right)$ and consider the sets

$$
A=\left\{x_{j} \mid j \geqslant j_{0}\right\} \cup\left\{x_{0}\right\} \text { and } B=\left\{x_{j} \mid j \geqslant j_{0}\right\} \cup\left\{y_{0}\right\} \text {. }
$$

The sections $S_{1}$ and $S_{2}$ determine that $A<B$. But the sections $S_{1}^{\prime}, S_{2}^{\prime}$ determine $B^{\prime}<A^{\prime}$, and $h$ preserves order, so also $B<A$. This contradicts Proposition 2.2 and hence the proof is complete.

5.6. Let $\left\{S_{n}^{\prime} \mid n \in \mathbf{Z}^{+}\right\}$be a covering system of cross-sections of $\left(M^{\prime}, \phi^{\prime}\right)$. Then there is a system $\left\{S_{n} \mid n \in \mathbf{Z}^{+}\right\}$of cross-sections of $(M, \phi)$ with $\pi\left(S_{n}\right)=T_{n}\left(n \in \mathbf{Z}^{+}\right)$and

$$
\operatorname{sgn} f_{i j}(x)=\operatorname{sgn} f_{i j}^{\prime}\left(x^{\prime}\right) \quad\left(x \in A_{i j}, i, j \in \mathbf{Z}^{+}\right) .
$$

Proof. We first note that for each $n \in \mathbf{Z}^{+}, \pi: \pi^{-1}\left(T_{n}\right) \rightarrow T_{n}$ is the projection of a locally trivial fiber bundle over the disk $T_{n}$, so there is a continuous lifting $\alpha_{n}$ : $T_{n} \rightarrow M$ with $\pi \circ \alpha_{n}=1$.

We now proceed by induction, taking any continuous lifting $\alpha_{1}: T_{1} \rightarrow M$ of $\pi$, and setting $\alpha_{1}\left(T_{1}\right)=S_{1}$. Suppose that we have constructed liftings $\alpha_{j}: T_{j} \rightarrow M$, and corresponding cross-sections $S_{j}=\alpha_{j}\left(T_{j}\right)$, for $j \leqslant n-1$, satisfying

$\left(\mathrm{c}_{n-1}\right) \quad \operatorname{sgn} f_{i j}(x)=\operatorname{sgn} f_{i j}^{\prime}\left(h^{-1} x\right) \quad\left(x \in A_{i j} ; i, j \leqslant n-1\right)$.

(Note that $\left(\mathrm{c}_{n-1}\right)$ implies that the $\left\{S_{j} \mid j \leqslant n-1\right\}$ are pairwise disjoint.) We want to construct $\alpha_{n}: T_{n} \rightarrow M, S_{n}=\alpha_{n}\left(T_{n}\right)$, so that

$$
\operatorname{sgn} f_{n j}(x)=\operatorname{sgn} f_{n j}^{\prime}\left(h^{-1} x\right) \quad\left(x \in A_{n j} ; j \leqslant n-1\right) .
$$


Let $\alpha_{n}: T_{n} \rightarrow M$ be an arbitrary lifting of $\pi, S_{n}=\alpha_{n}\left(T_{n}\right)$. Of course $S_{n}$ may not be disjoint from the $S_{j}(j \leqslant n-1)$, but at this stage it will be used only for reference. We must now modify $\alpha_{n}, S_{n}, f_{n j}$ to satisfy (*); we then relabel, so that the appropriately modified lifting, cross-section, and time maps are again denoted by $\alpha_{n}$, $S_{n}$, and $f_{n j}$ respectively. Note that if $(*)$ is satisfied, then $S_{n}$ is disjoint from the $S_{j}$ $(j \leqslant n-1)$.

For each $j \leqslant n-1$, define $f_{n j}^{+}, f_{n j}^{-}: T_{n} \rightarrow[-\infty, \infty]$ as follows:

$$
\begin{aligned}
& f_{n j}^{+}(x)= \begin{cases}f_{n j}(x) & \text { if } x \in T_{n} \cap T_{j} \text { and } f_{n j}^{\prime}\left(x^{\prime}\right)>0, \\
\infty & \text { otherwise; }\end{cases} \\
& f_{n j}^{-}(x)= \begin{cases}f_{n j}(x) & \text { if } x \in T_{n} \cap T_{j} \text { and } f_{n j}^{\prime}\left(x^{\prime}\right)<0, \\
-\infty & \text { otherwise. }\end{cases}
\end{aligned}
$$

5.7. For each $j, f_{n j}^{+}$is lower semicontinuous (lsc), and $f_{n j}^{-}$is upper semicontinuous (usc).

We show that $f_{n j}^{+}$is lsc, the verification that $f_{n j}^{-}$is usc being analogous. Thus fix $\alpha \in(-\infty, \infty)$; we must verify that $G_{\alpha}=\left\{x \in T_{n} \mid f_{n j}^{+}(x)>\alpha\right\}$ is open. Fix $x_{0} \in G_{\alpha}$. We consider separately the cases $f_{n j}^{+}\left(x_{0}\right)=\infty$ and $f_{n j}^{+}\left(x_{0}\right)<\infty$.

Case $1^{0}\left(f_{n j}^{+}\left(x_{0}\right)=\infty\right)$. If $x_{0}$ is not an interior point of $G_{\alpha}$, we can choose a sequence $\left\{x_{k}\right\}$ in $T_{n}$ with $\left\{x_{k}\right\} \rightarrow x_{0}$ and $f_{n j}^{+}\left(x_{k}\right) \leqslant \alpha$ for all $k$. We assert that $\lim _{k \rightarrow \infty} f_{n j}^{\prime}\left(x_{k}^{\prime}\right)=\infty$. For by definition of $f_{n j}^{+}, f_{n j}^{\prime}\left(x_{k}^{\prime}\right)$ is positive; also the possibility that $\left\{f_{n j}^{\prime}\left(x_{k}^{\prime}\right)\right\}$ has a bounded subsequence leads to a contradiction as follows. If $\lim _{l \rightarrow \infty} f_{n j}^{\prime}\left(x_{l}^{\prime}\right)=t^{\prime}$, then

$$
\phi^{\prime}\left(\alpha_{n}^{\prime}\left(x_{0}^{\prime}\right), t^{\prime}\right)=\lim _{l \rightarrow \infty} \phi^{\prime}\left(\alpha_{n}^{\prime}\left(x_{l}^{\prime}\right), f_{n j}^{\prime}\left(x_{l}^{\prime}\right)\right)=\lim _{l \rightarrow \infty} \alpha_{j}^{\prime}\left(x_{l}^{\prime}\right)
$$

is in $S_{j}^{\prime}$, so $x_{0}^{\prime} \in T_{j}^{\prime} \cap T_{n}^{\prime}$ and $f_{n j}^{\prime}\left(x_{0}^{\prime}\right)=t^{\prime}>0$; hence $f_{n j}^{+}\left(x_{0}\right)=f_{n j}\left(x_{0}\right)$, contrary to our case assumption $f_{n j}^{+}\left(x_{0}\right)=\infty$. But if $\lim _{k \rightarrow \infty} f_{n j}^{\prime}\left(x_{k}^{\prime}\right)=\infty$, then by Lemma 5.5 also

$$
\lim _{k \rightarrow \infty} f_{n j}^{+}\left(x_{k}\right)=\lim _{k \rightarrow \infty} f_{n j}\left(x_{k}\right)=+\infty,
$$

and this is impossible since we chose $\left\{x_{k}\right\}$ so $f_{n j}^{+}\left(x_{k}\right) \leqslant \alpha$. This contradiction shows that $x_{0}$ is an interior point of $G_{\alpha}$ in case $f_{n j}^{+}\left(x_{0}\right)=\infty$.

Case $2^{0}\left(f_{n j}^{+}\left(x_{0}\right)<\infty\right)$. Here the fact that $x_{0}$ is an interior point of $G_{\alpha}$ follows immediately from the continuity of $f_{n j}$ on its domain $T_{n} \cap T_{j}$.

5.8. Now define functions $u_{n}, l_{n}: T_{n} \rightarrow[-\infty, \infty]$ by

$$
u_{n}=\min \left\{f_{n j}^{+} \mid j \leqslant n-1\right\} ; \quad l_{n}=\max \left\{f_{n j}^{-} \mid j \leqslant n-1\right\} .
$$

Then $u_{n}$ is $l s c, l_{n}$ is usc, and for all $x \in T_{n}$ we have $l_{n}(x)<u_{n}(x)$.

The semicontinuity of $u_{n}, l_{n}$ follows immediately from 5.7. To check that $l_{n}<u_{n}$ at $x \in T_{n}$ we may as well assume both are finite, hence that $u_{n}(x)=f_{n j}(x)$ and $l_{n}(x)=f_{n i}(x)$ for some $i, j \leqslant n-1$. Note that then $f_{n j}^{\prime}\left(x^{\prime}\right)>0, f_{n i}^{\prime}\left(x^{\prime}\right)<0$ (by our definitions of $f_{n j}^{+}$and $\left.f_{n j}^{-}\right)$. Hence $f_{i j}^{\prime}\left(x^{\prime}\right)=f_{n j}^{\prime}\left(x^{\prime}\right)-f_{n i}^{\prime}\left(x^{\prime}\right)>0$. By induction, $\operatorname{sgn} f_{i j}(x)=\operatorname{sgn} f_{i j}^{\prime}\left(x^{\prime}\right)$, so

$$
u_{n}(x)-l_{n}(x)=f_{n j}(x)-f_{n i}(x)=f_{i j}(x)>0,
$$

as asserted. 
It is now possible to modify $S_{n}$ so that $(*)$ is satisfied. By the Separation Lemma 5.4, there is a continuous function $t: T_{n} \rightarrow \mathbf{R}^{1}$ with

$$
l_{n}(x)<t(x)<u_{n}(x) \quad\left(x \in T_{n}\right) .
$$

We replace $\alpha_{n}$ with $\hat{\alpha}_{n}: T_{n} \rightarrow M$ defined by

$$
\hat{\alpha}_{n}(x)=\phi\left(\alpha_{n}(x), t(x)\right),
$$

set $\hat{S}_{n}=\hat{\alpha}_{n}\left(T_{n}\right)$, and define $\hat{f}_{n j}$ by $\phi\left(\hat{\alpha}_{n}(x), \hat{f}_{n j}(x)\right)=\alpha_{j}(x)$. It is easily checked that these adjusted time maps satisfy

$$
\operatorname{sgn} \hat{f}_{n j}(x)=\operatorname{sgn} f_{n j}^{\prime}\left(x^{\prime}\right), \quad x \in T_{n}, j \leqslant n-1 .
$$

Finally we relabel: $S_{n}=\hat{S}_{n}, \alpha_{n}=\hat{\alpha}_{n}, f_{n j}=\hat{f}_{n j}, f_{j n}=-\hat{f}_{n j}(j \leqslant n-1)$. This establishes the induction step, so the proof of 5.6 is complete.

We now must adjust the system $\left\{S_{n} \mid n \in \mathbf{Z}^{+}\right\}$of cross-sections obtained in 5.6 to obtain local finiteness. Recall that each $S_{n}$ is homeomorphic with the closed disk $D^{m-1}$; we use $\partial S_{n}$ to denote the boundary of this disk, and Int $S_{n}=S_{n} \backslash \partial S_{n}$ to denote its (relative) interior. We first establish that the system $\left\{S_{n}\right\}$ admits a "shrinking", viz.:

5.9. Shrinking Lemma. There is a system of cross-sections $\left\{\tilde{S}_{n} \mid n \in \mathbf{Z}^{+}\right\}$such that $\tilde{S}_{n} \subseteq$ Int $S_{n}$ for all $n \in \mathbf{Z}^{+}$and $M / \phi=\bigcup\left\{\pi\left(\operatorname{Int} \tilde{S}_{n}\right) \mid n \in \mathbf{Z}^{+}\right\}$.

Proof. We proceed by induction. For each $x \in \partial S_{1}$ there is a $j \geqslant 2$ and a cross-section $S_{j}^{1} \subseteq \operatorname{Int} S_{j}$ such that $x \in\left(\right.$ Int $\left.S_{j}^{1}\right) \cdot \mathbf{R}^{1}$. Since $\partial S_{1}$ is compact, there is a finite set $J_{1} \subseteq \mathbf{Z}^{+}$such that

$$
\left(\partial S_{1}\right) \cdot \mathbf{R}^{1} \subseteq \bigcup_{j \in J_{1}}\left(\operatorname{Int} S_{j}^{1}\right) \cdot \mathbf{R}^{1}
$$

Choose $\tilde{S}_{1} \subseteq$ Int $S_{1}$ so that

$$
S_{1} \cdot \mathbf{R}^{1} \subseteq\left(\operatorname{Int} \tilde{S}_{1}\right) \cdot \mathbf{R}^{1} \cup \bigcup_{j \in J_{1}}\left(\operatorname{Int} S_{j}^{1}\right) \cdot \mathbf{R}^{1} .
$$

We remark that, for each $j \in J_{1}$, when we later construct $\tilde{S}_{j}$, we will impose the restriction $S_{j}^{1} \subseteq \tilde{S}_{j}$.

Suppose now that we have constructed cross-sections $\tilde{S}_{1}, \ldots, \tilde{S}_{n}$ with $\tilde{S}_{k} \subseteq$ Int $S_{k}$ $(k \leqslant n)$, finite sets $J_{k} \subseteq\left\{l \in \mathbf{Z}^{+} \mid l>k\right\}(k \leqslant n)$, and cross-sections $s_{j}^{k}\left(j \in J_{k}\right.$, $k \leqslant n$ ) satisfying

(a) for each $k \leqslant n, S_{k} \cdot \mathbf{R}^{1} \subseteq \bigcup_{j \leqslant k}\left(\right.$ Int $\left.\tilde{S}_{j}\right) \cdot \mathbf{R}^{1} \cup \bigcup_{j \in J_{k}}\left(\right.$ Int $\left.S_{j}^{k}\right) \cdot \mathbf{R}^{1}$ and

(b) for any $l \leqslant j \leqslant n$, if $j \in J_{l}$, then $S_{j}^{l} \subseteq \tilde{S}_{j}$.

Now consider $S_{n+1}$. Choose a finite set $J_{n+1} \subseteq\left\{l \in \mathbf{Z}^{+} \mid l>n+1\right\}$ and sections $S_{j}^{n+1} \subseteq$ Int $S_{j}$ for each $j \in J_{n+1}$ so that

$$
\left(\partial S_{n+1}\right) \cdot \mathbf{R}^{1} \subseteq \bigcup_{j \leqslant n}\left(\operatorname{Int} \tilde{S}_{j}\right) \cdot \mathbf{R}^{1} \cup \bigcup_{j \in J_{n+1}}\left(\operatorname{Int} S_{j}^{n+1}\right) \cdot \mathbf{R}^{1} .
$$

(We may see that this is possible as follows. Fix $x \in \partial S_{n+1}$. If $x \in\left(\operatorname{Int} S_{j}\right) \cdot \mathbf{R}^{1}$ for some $j>n+1$, choose $S_{j}^{n+1}$ with $x \in\left(\right.$ Int $\left.S_{j}^{n+1}\right) \cdot \mathbf{R}^{1}$ and $S_{j}^{n+1} \subseteq \operatorname{Int} S_{j}$. If $x$ is in no $\left(\right.$ Int $\left.S_{j}\right) \cdot \mathbf{R}^{1}$ for $j>n+1$, then choose $k \leqslant n$ with $x \in\left(\right.$ Int $\left.S_{k}\right) \cdot \mathbf{R}^{1}$. Now by (a) 
and (b), we have

$$
\begin{aligned}
x & \in\left(\operatorname{Int} S_{k}\right) \cdot \mathbf{R}^{1} \subseteq \bigcup_{j \leqslant k}\left(\operatorname{Int} \tilde{S}_{j}\right) \cdot \mathbf{R}^{1} \cup \bigcup_{j \in J_{k}}\left(\operatorname{Int} S_{j}^{k}\right) \cdot \mathbf{R}^{1} \\
& \subseteq \bigcup_{j \leqslant k}\left(\operatorname{Int} \tilde{S}_{j}\right) \cdot \mathbf{R}^{1} \cup \bigcup_{\substack{j \in J_{k} \\
j \leqslant n}}\left(\operatorname{Int} S_{j}\right) \cdot \mathbf{R}^{1} \cup \bigcup_{\substack{j \in J_{k} \\
j \geqslant n+1}}^{\bigcup}\left(\operatorname{Int} S_{j}\right) \cdot \mathbf{R}^{1}
\end{aligned}
$$

so $x \in \bigcup_{j \leqslant n}\left(\right.$ Int $\left.\tilde{S}_{j}\right) \cdot \mathbf{R}^{1}$.) Now choose $\tilde{S}_{n+1} \subseteq \operatorname{Int} S_{n+1}$ so that (a) and (b) hold with $n+1$ with $n+1$ in place of $n$.

By induction we obtain a system $\left\{\tilde{S}_{n} \mid n \in \mathbf{Z}^{+}\right\}$with $\tilde{S}_{n} \subseteq$ Int $S_{n}$ for all $n \in \mathbf{Z}^{+}$, and such that (a) and (b) hold for all $n$. We must check that any $x \in M$ is contained in some $\left(\right.$ Int $\left.\tilde{S}_{n}\right) \cdot \mathbf{R}^{1}$. Choose $n$ with $x \in\left(\operatorname{Int} S_{n}\right) \cdot \mathbf{R}^{1}$. But

$$
\left(\text { Int } S_{n}\right) \cdot \mathbf{R}^{1} \subseteq \bigcup_{j \leqslant n}\left(\operatorname{Int} \tilde{S}_{j}\right) \cdot \mathbf{R}^{1} \cup \bigcup_{j \in J_{n}}\left(\operatorname{Int} S_{j}^{n}\right) \cdot \mathbf{R}^{1},
$$

and by (b) $S_{j}^{n} \subseteq \tilde{S}_{j}$ for any $j \in J_{n}$. Therefore, if $m=\max J_{n}$, we have $x \in$ $\bigcup_{j \leqslant m}\left(\right.$ Int $\left.\tilde{S}_{j}\right) \cdot \mathbf{R}^{1}$ as desired.

5.10. REMARK. We emphasize that we have not assumed that $S_{n}$ is locally finite in 5.9. This accounts for the fact that the preceding proof is more complicated than the usual proof of the "shrinking theorem" for point-finite covers of normal spaces; compare [Du, Theorem 6.1].

5.11. Construction OF COMPATIBLE COVERING SYSTEMS. We can now complete the proof of Proposition 5.2. Again $\left\{S_{n}^{\prime}\right\}$ denotes a given covering system of cross-sections of $\left(M^{\prime}, \phi^{\prime}\right)$, and $\left\{S_{n}\right\}$ is the system of cross-sections constructed in 5.6. Let $\left\{\tilde{S}_{n}\right\}$ be the shrinking of $\left\{S_{n}\right\}$ as in 5.9. Choose a sequence $\left\{\varepsilon_{n}^{\prime}\right\}$ of positive reals so that $\lim _{n \rightarrow \infty} \varepsilon_{n}^{\prime}=0$ and such that $\left\{S_{n}^{\prime} \cdot\left[-\varepsilon_{n}^{\prime}, \varepsilon_{n}^{\prime}\right]\right\}$ is a pairwise disjoint collection. We construct inductively a (locally finite) covering system $\left\{R_{i}\right\}$ of $(M, \phi)$ so that for each $i \in \mathbf{Z}^{+}$, there exist $n \in \mathbf{Z}^{+}$and $t_{i} \in\left[\varepsilon_{n}^{\prime}, \varepsilon_{n}^{\prime}\right]$ with $R_{i} \subseteq S_{n} \cdot t_{i}$.

Let $Q_{1}=R_{1}=S_{1}$. Suppose that we have determined integers $1=m_{1}<m_{2}<$ $\cdots<m_{n}$ and cross-sections $Q_{i} \cong D^{m-1}$ for each $i \leqslant m_{n}$ such that

(a) for each $k \leqslant n$ and $i \in I_{k}=\left\{m_{k-1}+1, \ldots, m_{k}\right\}, Q_{i} \subseteq S_{k}$;

(b) for each $k \leqslant n, \bigcup_{j \leqslant k}\left(\right.$ Int $\left.S_{j}\right) \cdot \mathbf{R}^{1} \bigcup_{i \leqslant m_{k}}\left(\right.$ Int $\left.Q_{i}\right) \cdot \mathbf{R}^{\mathbf{1}}$; and

(c) for each $k \leqslant n, i \leqslant m_{k-1}$ and $j \in I_{k}$ we have either

$$
Q_{j} \cap\left(Q_{i}^{k} \cdot \mathbf{R}^{1}\right)=\varnothing \text { or }\left|g_{i j}(x)\right|>k \text { for all } x \in Q_{j} \cap\left(Q_{i}^{k} \cdot \mathbf{R}^{1}\right) .
$$

Here $Q_{i}^{k}$ denotes the subset of $Q_{i}$ that corresponds to the closed disk of radius $1-1 / k$ under the homeomorphisms $Q_{i} \cong D^{m-1}$, and $g_{i j}(x)$ denotes the time along the $\phi$-orbit $\gamma(x)$ from $Q_{i}$ to $Q_{j}$. Note that if $i \in I_{k}$ and $j \in I_{l}$, then

$$
\operatorname{sgn} g_{i j}(x)=\operatorname{sgn} f_{k l}^{\prime}\left(h^{-1} \pi(x)\right), \quad x \in Q_{j} \cap\left(Q_{i} \cdot \mathbf{R}^{1}\right) .
$$

This applies even if $k=l$, in which case both sides are zero; i.e. for $i, j \in I_{k}, Q_{i}$ and $Q_{j}$ may not be disjoint (they will be made disjoint after the inductive construction of the $Q_{i}$ is completed; the resulting sections will be the $R_{i}$ ). 
Now consider $S_{n+1}$. Let $O=S_{n+1} \cap \bigcup_{i \leqslant m_{n}}$ (Int $\left.Q_{i}\right) \cdot \mathbf{R}^{1}$. We assert that for each $x \in \tilde{S}_{n+1} \backslash O$ there is an $(m-1)$ disk $Q_{x} \subseteq S_{n+1}$ with $x \in$ Int $Q_{x}$, and such that whenever $Q_{x} \cap Q_{i}^{n+1} \cdot \mathbf{R}^{1} \neq \varnothing$ for $i \leqslant m_{n}$, we have

$$
\left|g_{i}(y)\right|>n+1 \text { for all } y \in Q_{x} \cap\left(Q_{i}^{n+1} \cdot \mathbf{R}^{1}\right),
$$

where $g_{i}(y)$ denotes the time along the $\phi$-orbit $\gamma(y)$ from $S_{n+1}$ to $Q_{i}$. To see this, fix $x \in \tilde{S}_{n+1} \backslash O$ and $i \leqslant m_{n}$. Let $A_{i}=S_{n+1} \cap\left(Q_{i} \cdot \mathbf{R}^{1}\right)$, the domain of $g_{i}$. If $x \in$ $\overline{A_{i}} \backslash A_{i}$, then by 5.5 there is a neighborhood $N_{i}$ in $S_{n+1}$ of $x$ such that

$$
\left|g_{i}(y)\right|>n+1 \text { for all } y \in N_{i} \cap A_{i} \text {. }
$$

If $x \notin \overline{A_{i}}$, then let $N_{i}$ be a neighborhood in $S_{n+1}$ of $x$ that does not intersect $S_{n+1} \cap Q_{i}^{n+1} \cdot \mathbf{R}^{1} \subseteq A_{i}$. Since $x \notin O$, the only remaining possibility is that $x \in S_{n+1}$ $\cap\left(\partial Q_{i}\right) \cdot \mathbf{R}^{1}$, and again some neighborhood $N_{i}$ of $x$ in $S_{n+1}$ misses $S_{n+1} \cap Q_{i}^{n+1} \cdot \mathbf{R}^{1}$. Hence we may take $Q_{x}$ to be an $(m-1)$ disk contained in $\bigcap_{i \leqslant m_{n}} N_{i}$.

Now the (relative) interiors of finitely many of these $Q_{x}$ cover $\tilde{S}_{n+1} \backslash O$; we label these as $Q_{m_{n}+1}, Q_{m_{n}+2}, \ldots, Q_{m_{n+1}}$. By our construction, $Q_{1}, Q_{2}, \ldots, Q_{m_{n+1}}$ now satisfy (a)-(c) with $(n+1)$ in place of $n$.

Thus we obtain by induction a family $Q_{i}$ of cross-sections of $(M, \phi)$, satisfying (a)-(c) for all $n$. It follows from (b) that $M / \phi=\bigcup_{i \in \mathbf{Z}^{+}} \pi$ (Int $Q_{i}$ ). We now check that $\left\{Q_{i}\right\}$ is locally finite. Fix $x \in M$, choose $i \in \mathbf{Z}^{+}$with $x \in\left(\right.$ Int $\left.Q_{i}\right) \cdot \mathbf{R}^{1}$ and choose $N \in \mathbf{Z}^{+}$so large that $i<m_{N}$ and $x$ is an interior point of the flow box $B=Q_{i}^{N} \cdot[-N, N]$. We assert that only finitely many of the $Q_{j}$ meet $B$. In fact if $j>m_{N}$ (so $Q_{j}$ was constructed at some stage $n>N$ of our induction) then by (c) either $Q_{j}$ misses $Q_{i}^{N} \cdot \mathbf{R}^{1}$ or $\left|g_{i j}(y)\right|>N$ for all $y \in Q_{j} \cap Q_{i}^{N} \cdot \mathbf{R}^{1}$. In either case $Q_{j} \cap B=\varnothing$. Also note that from our construction, for arbitrary $i \in I_{k}$ and $j \in I_{l}$ we have

$$
\operatorname{sgn} g_{i j}(x)=\operatorname{sgn} f_{k l}^{\prime}\left(h^{-1} \pi x\right) \quad\left(x \in Q_{i} \cap\left(Q_{j} \cdot \mathbf{R}^{1}\right)\right) .
$$

We now define $R_{i}$ by translating the $Q_{i}$ along the flow to obtain disjointness; this is possible as follows. Since $\left\{Q_{i}\right\}$ is locally finite, we may choose a positive sequence $\left\{\varepsilon_{n}\right\}$ with $\varepsilon_{n} \leqslant \varepsilon_{n}^{\prime}, \lim _{n \rightarrow \infty} \varepsilon_{n}=0$, and so that the sets $\left(\bigcup_{i \in I_{k}} Q_{i}\right) \cdot\left[-\varepsilon_{k}, \varepsilon_{k}\right]$ for $k \in \mathbf{Z}^{+}$are pairwise disjoint. Now for each $k \in \mathbf{Z}^{+}$choose distinct $t_{i} \in\left[-\varepsilon_{k}, \varepsilon_{k}\right]$ for $i \in I_{k}$ and set $R_{i}=Q_{i} \cdot t_{i}$. Then $\left\{R_{i} \mid i \in \mathbf{Z}^{+}\right\}$is a covering system for $(M, \phi)$.

Finally we define a covering system $\left\{R_{i}^{\prime} \mid i \in \mathbf{Z}^{+}\right\}$for $\left(M^{\prime}, \phi^{\prime}\right)$ by

$$
R_{i}^{\prime}=\alpha_{k}^{\prime}\left(h^{-1} \pi\left(R_{i}\right)\right) \cdot t_{i}
$$

for each $k \in \mathbf{Z}^{+}$and $i \in I_{k}$. It is easily verified that $\left\{R_{i}\right\}$ and $\left\{R_{i}^{\prime}\right\}$ are compatible.

6. Complete systems of cross-sections. We will need one further refinement of our notion of covering systems of cross-sections in the proof of the reparametrization theorem below.

6.1. Definitions. Let $(M, \phi)$ be a completely unstable dynamical system and let $\left\{S_{n} \mid n \in \mathbf{Z}^{+}\right\}$be a covering system of cross-sections. We say that $\left\{S_{n}\right\}$ is complete if for each $x \in M, x \cdot(0, \infty)$ meets some Int $S_{j}$ and $x \cdot(-\infty, 0)$ meets some Int $S_{k}$.

If $\left\{S_{n}\right\}$ and $\left\{R_{n}\right\}$ are systems of cross-sections for $(M, \phi)$ and $\left\{S_{n}\right\} \subseteq\left\{R_{n}\right\}$, we say $\left\{R_{n}\right\}$ augments $\left\{S_{n}\right\}$. 
6.2. Lemma. Let $(M, \phi)$ and $\left(M^{\prime}, \phi^{\prime}\right)$ be $C^{r}$ completely unstable dynamical systems and let $h: M^{\prime} / \phi^{\prime} \rightarrow M / \phi$ be an order preserving homeomorphism. If $\left\{S_{n} \mid n \in \mathbf{Z}^{+}\right\}$and $\left\{S_{n}^{\prime} \mid n \in \mathbf{Z}^{+}\right\}$are compatible covering systems of cross-sections for $(M, \phi)$ and $\left(M^{\prime}, \phi^{\prime}\right)$ respectively, then they can be augmented to compatible complete systems.

Proof. We proceed by induction. Assume that we have determined integers $k_{1}<k_{2}<\cdots<k_{m-1}$, positive numbers $t_{1}<t_{2}<\cdots<t_{m-1}$, and cross-sections $\left\{R_{j} \mid j \leqslant k_{m-1}\right\},\left\{R_{j}^{\prime} \mid j \leqslant k_{m-1}\right\}$ satisfying the following (where $f_{i j}, f_{i j}^{\prime}$ are as above, $g_{i j}: \pi\left(S_{i}\right) \cap \pi\left(R_{j}\right) \rightarrow \mathbf{R}^{1}$ denotes the time from $S_{i}$ and $R_{j}$, to $g_{i j}^{\prime}$ is defined analogously):

(a) At each stage $l \leqslant m-1, t_{l}>l$ is chosen so that for each $i \leqslant l$ and $j \in \mathbf{Z}^{+}$we have

$$
\left|f_{i j}(x)\right|>t_{l} \text { implies }\left|f_{i j}^{\prime}\left(h^{-1} x\right)\right|>l ;
$$

for each $i<l$ and $j \leqslant k_{l-1}$ we have

$$
\left|g_{i j}(x)\right|>t_{l} \text { implies }\left|g_{i j}^{\prime}\left(h^{-1} x\right)\right|>l \text {. }
$$

(b) At each stage $l \leqslant m-1$, for any $p \in \cup_{i \leqslant l} S_{i}$ there exist integers $j, k \in$ $\left(k_{l-1}, k_{l}\right]$ and times $r_{p}>t_{l}, s_{p}<-t_{l}$ with $p \cdot r_{p} \in R_{j}, p \cdot s_{p} \in R_{k}$; analogously for the $R_{j}^{\prime}, j=k_{l-1}+1, \ldots, k_{l}$.

(c) At each stage $l \leqslant m-1,\left\{S_{n} \mid n \in \mathbf{Z}^{+}\right\} \cup\left\{R_{j} \mid j \leqslant k_{l}\right\}$ is compatible with $\left\{S_{n}^{\prime} \mid n \in \mathbf{Z}_{+}\right\} \cup\left\{R_{j}^{\prime} \mid j \leqslant k_{l}\right\}$.

We now proceed to extend the augmentations of $\left\{S_{i}\right\}$ and $\left\{S_{i}^{\prime}\right\}$ to stage $m$. For $x \in M / \phi$ let $x^{\prime}$ denote $h^{-1}(x) \in M^{\prime} / \phi^{\prime}$; set

$$
C_{m}^{\prime}=\left[\bigcup_{i \leqslant m} S_{i}^{\prime}\right] \cdot[-m, m] .
$$

(1) There exists $t_{m}>\max \left(t_{m-1}, m\right)$ such that for each $i \leqslant m$ and $j \in \mathbf{Z}^{+}$

$$
\left|f_{i j}(x)\right|>f_{m} \text { implies }\left|f_{i j}^{\prime}\left(x^{\prime}\right)\right|>m
$$

and for each $i \leqslant m$ and $j \leqslant k_{m-1}$

$$
\left|g_{i j}(x)\right|>t_{m} \text { implies }\left|g_{i j}^{\prime}\left(x^{\prime}\right)\right|>m \text {. }
$$

For if no such $t_{m}$ exists, then there is a sequence $\left\{x_{k}\right\} \subseteq \bigcup_{i \leqslant m} \pi\left(S_{i}\right)$ and a sequence $\left\{j_{k}\right\} \subseteq \mathbf{Z}^{+}$such that $\left|f_{i j_{k}}\left(x_{k}\right)\right| \rightarrow+\infty$ but $\left|f_{i j_{k}}^{\prime}\left(x_{k}^{\prime}\right)\right| \leqslant m$. Without loss of generality we may assume $\left\{x_{k}\right\} \subseteq \pi\left(S_{1}\right)$, that $x_{k} \rightarrow x \in \pi\left(S_{1}\right)$, and that $j_{k}=j$ (fixed) for all $k \in \mathbf{Z}^{+}$. The last of these requirements can be met because $\left\{S_{j}^{\prime}\right\}$ is locally finite so only finitely many of the $S_{j}^{\prime}$ meet the compact set $C_{m}^{\prime}$. But then

$$
\left|f_{1 j}\left(x_{k}\right)\right| \rightarrow+\infty \text { while }\left|f_{1 j}^{\prime}\left(x_{k}^{\prime}\right)\right| \leqslant m,
$$

contrary to Lemma 5.5. The assertion on the $g_{i j}$ may be proved similarly.

Now choose $t_{m}$ as in (1) above and define

$$
C_{m}=\left[\bigcup_{i \leqslant m} S_{i}\right] \cdot\left[-t_{m}, t_{m}\right] .
$$


For a disk $D_{p}\left(\cong D^{m-1}\right)$ in $S_{i}$, let $D_{p}^{\prime}$ denote the corresponding disk $\left(\pi^{\prime-1} h^{-1} \pi\left(D_{p}\right)\right.$ $\left.\cap S_{i}^{\prime}\right)$ in $S_{i}^{\prime}$; analogously for a point $p \in S_{i}$ let $p^{\prime}=\left(\pi^{\prime-1} h^{-1} \pi(p) \cap S_{i}^{\prime}\right)$. Define

$$
W=\left(\bigcup_{i \geqslant 1} S_{i}\right) \cup\left(\bigcup_{j \leqslant k_{m-1}} R_{j}\right) \cup C_{m}
$$

and

$$
W^{\prime}=\left(\bigcup_{i \geqslant 1} S_{i}^{\prime}\right) \cup\left(\bigcup_{j \leqslant k_{m-1}} R_{j}^{\prime}\right) \cup C_{m}^{\prime} .
$$

(2) For each $p \in S_{1}$, there exists a disk neighborhood $D_{p}\left(\cong D^{m-1}\right)$ of $p$ in $S_{1}$, and there are times $r_{p}>t_{m}, r_{p}^{\prime}>m$ such that

( $\alpha) D_{p} \cdot r_{p} \cap W=\varnothing$;

( $\beta$ ) $D_{p}^{\prime} \cdot r_{p}^{\prime} \cap W^{\prime}=\varnothing$;

( $\gamma) D_{p} \cdot r_{p}$ is related to $\left\{S_{i} \mid i \geqslant 1\right\} \cup\left\{R_{j} \mid j \leqslant k_{m-1}\right\}$ exactly as $D_{p}^{\prime} \cdot r_{p}^{\prime}$ is related to $\left\{S_{i}^{\prime} \mid i \geqslant 1\right\} \cup\left\{R_{j}^{\prime} \mid j \leqslant k_{m-1}\right\}$; viz., the $\phi$ orbit $\gamma(p)$ meets $S_{i}\left(R_{j}\right)$ ahead of $D_{p} \cdot r_{p}$ if and only if the corresponding $\phi^{\prime}$ orbit $\pi^{-1} h^{-1} \pi(p)$ meets $S_{i}^{\prime}$ ( $R_{j}^{\prime}$ respectively) ahead of $D_{p}^{\prime} \cdot r_{p}^{\prime}$.

We may see this as follows. For $p \in S_{1}$ first choose $r_{p}>t_{m}$ so that $p \cdot r_{p} \notin W$. By our choice of $t_{m}$, we can then choose $r_{p}^{\prime}>m$ so that $p^{\prime} \cdot r_{p}^{\prime} \notin W^{\prime}$ and $p^{\prime} \cdot r_{p}^{\prime}$ is related to the $S_{i}^{\prime}\left(R_{j}^{\prime}\right)$ just as $p \cdot r_{p}$ is related to the $S_{i}\left(R_{j}\right)$. It follows that there are neighborhoods $U_{p} \subseteq M, U_{p}^{\prime} \subseteq M^{\prime}$ such that

(i) $p \cdot r_{p} \in U_{p}, U_{p} \cap W=\varnothing$;

(ii) $p^{\prime} \cdot r_{p}^{\prime} \in U_{p}^{\prime}, U_{p}^{\prime} \cap W^{\prime}=\varnothing$; and

(iii) $U_{p}^{\prime}$ is related to the $S_{i}^{\prime}\left(R_{j}^{\prime}\right)$ as $U_{p}$ is related to the $S_{i}\left(R_{j}\right)$.

Finally choose a disk neighborhood $D_{p}\left(\cong D^{m-1}\right)$ of $p$ so small that $D_{p} \cdot r_{p} \subseteq U_{p}$ and $D_{p}^{\prime} \cdot r_{p}^{\prime} \subseteq U_{p}^{\prime}$; the resulting disks satisfy all the assertions of (2).

Since $S_{1}$ is compact, finitely many $\stackrel{\circ}{D}_{p}$ cover it, say those with $p=p_{1}, \ldots, p_{h}$. Note that we may assume each of $\left\{D_{p_{i}} \cdot r_{p_{i}} \mid i \leqslant h\right\}$ and $\left\{D_{p_{i}}^{\prime} \cdot r_{p_{i}}^{\prime} \mid i \leqslant h\right\}$ is a disjoint collection, by adjusting slightly if necessary the times $r_{p_{i}}, r_{p_{i}}^{\prime}$. Analogously we can obtain disjoint collections $\left\{E_{p_{i}} \cdot s_{p_{i}} \mid h<i \leqslant k\right\}\left(\left\{E_{p_{i}}^{\prime} \cdot s_{p_{i}}^{\prime} \mid h<i \leqslant k\right\}\right)$ of disks below $C_{m}$ ( $C_{m}^{\prime}$ respectively), satisfying $(\alpha)-(\gamma)$ of (2). Here we may assume each $s_{p_{i}}<-t_{m}, s_{p_{i}}^{\prime}<-m$, and that $\left\{\stackrel{\circ}{E}_{p_{i}} \mid h<i \leqslant k\right\}$ covers $S_{1}$. We now relabel all the disks $D_{p_{i}} \cdot r_{p_{i}}, E_{p_{i}} \cdot s_{p_{i}}$ by $R_{k_{m-1}+1}, \ldots, R_{k_{m}^{\prime}}$ (so $k_{m}^{\prime}=k_{m-1}+k$ ); label the corresponding disks in $M^{\prime}$ by $R_{k_{m-1}+1}^{\prime \prime}, \ldots, R_{k_{m}^{\prime}}^{\prime \prime}$. At this point the $R_{i}^{\prime \prime}$ may fail to be related to each other in the same way the $R_{j}\left(k_{m-1}<j \leqslant k_{m}^{\prime}\right)$ are, but the argument of 5.6-5.8 shows that we can adjust the $R_{j}^{\prime \prime}$ to obtain appropriately related cross-sections $R_{j}^{\prime}\left(k_{m-1}<j \leqslant k_{m}^{\prime}\right)$, such that the $R_{j}^{\prime}$ also miss $C_{m}^{\prime}$. Observe that, by our construction, the collections $\left\{S_{i} \mid i \geqslant 1\right\} \cup\left\{R_{j} \mid j \leqslant k_{m}^{\prime}\right\}$ and $\left\{S_{i}^{\prime} \mid i \geqslant 1\right\} \cup\left\{R_{j}^{\prime} \mid j\right.$ $\left.\leqslant k_{m}^{\prime}\right\}$ satisfy the required conclusion (a)-(c) of our inductive construction, except that in (b) we have only $S_{1}$ in place of $\bigcup_{i \leqslant m} S_{i}$.

We must now repeat the argument from (2) on, replacing $S_{1}$ with $S_{2}, \ldots, S_{l}$ in succession, at each stage augmenting $W$ and $W^{\prime}$ by adding the sections $R_{j}, R_{j}^{\prime}$ constructed at the preceding stage. If we denote by $R_{i}, R_{i}^{\prime}\left(i=k_{m-1}+1, \ldots, k_{m}\right)$ all the new sections produced in this way, then the resulting systems satisfy (a)-(c) with $m$ in place of $m-1$, as is required. 
Thus we obtain by induction augmented systems $\left\{S_{i} \mid i \in \mathbf{Z}^{+}\right\} \cup\left\{R_{j} \mid j \in \mathbf{Z}^{+}\right\}$in $M$ and $\left\{S_{i}^{\prime} \mid i \in \mathbf{Z}^{+}\right\} \cup\left\{R_{j}^{\prime} \mid j \in \mathbf{Z}^{+}\right\}$in $M^{\prime}$. These are the required complete systems. Note that compatibility follows directly from the construction; also the augmented systems remain locally finite, since in fact each of the compact sets $C_{m}\left(C_{m}^{\prime}\right)$ meets only finitely many of the $R_{j}$ ( $R_{j}^{\prime}$ respectively). Hence only completeness remains to be checked. But suppose there is some $q \in M$ such that $q \cdot(0, \infty)$ does not meet any $S_{i}\left(i \in \mathbf{Z}^{+}\right)$(the argument is similar if $q \cdot(-\infty, 0)$ does not hit any $\left.S_{i}\right)$. Let $t \in(-\infty, 0]$ be such that $p=q \cdot t \in S_{l}$ for some $l \in \mathbf{Z}^{+}$. Let $R_{k}$ be a cross-section added at the $m$ th stage of our construction, where $m$ is chosen so $m \geqslant l, t_{m}>-t$, and $p \cdot(0, \infty) \cap \stackrel{\circ}{R}_{k} \neq \varnothing$. Since $R_{k}$ is added at stage $m$, we must have $g_{l k}(p)>t_{m}$ $>-t$. Thus if we set $t^{\prime}=g_{l k}(p)+t$, then $t^{\prime} \in(0, \infty)$ and in fact

$$
q \cdot t^{\prime}=(q \cdot t) \cdot g_{l k}(p)=p \cdot g_{l k}(p) \in \stackrel{\circ}{R}_{k},
$$

as desired. This completes the proof of 6.2.

6.3. RemarK. We remark that the complete system $\left\{S_{i} \mid i \in \mathbf{Z}^{+}\right\} \cup\left\{R_{j} \mid\right.$ for each $\left.i \in \mathbf{Z}^{+}, k_{l-1}<j \leqslant k_{1}\right\}$ of cross-sections obtained above can be constructed so that it admits a shrinking that is also complete. In fact when we construct the disks $D_{p} \cdot r_{p}$ in 6.2 , we can actually construct disks $D_{p} \cdot r_{p}$ and $\tilde{D}_{p} \cdot r_{p}$ with $\tilde{D}_{p} \subseteq$ Int $D_{p}$ and such that (for those added at the $m$ th stage) finitely many Int $\tilde{D}_{p}$ cover $\bigcup_{i \leqslant m} S_{i}$. Thus, if we denote the cross-sections $\tilde{D}_{p} \cdot r_{p}$ as $\tilde{R}_{j}$, then from Lemma 5.9, $\left\{\tilde{S}_{i} \mid i \in\right.$ $\left.\mathbf{Z}^{+}\right\} \cup\left\{\tilde{R}_{j} \mid\right.$ for each $\left.i \in \mathbf{Z}^{+}, k_{i-1}<j \leqslant k_{i}\right\}$ is the desired shrinking that is also complete.

\section{Reparametrization of completely unstable flows.}

7.1. Definition. If $\psi$ is a continuous flow on the $m$-manifold $M$, we say that the flow $\tilde{\psi}$ is a reparametrization of $\psi$ if the identity mapping $1: M \rightarrow M$ is a topological equivalence of $\psi$ with $\tilde{\psi}$.

We remark that we admit the possibility that $\tilde{\psi}$ is only $C^{0}$ even though $\psi$ may be smooth. In any case a third system $\left(M^{\prime}, \psi^{\prime}\right)$ is topologically equivalent to $(M, \psi)$ if and only if it is equivalent to $(M, \tilde{\psi})$.

The object of the present section is to prove the following reparametrization theorem. It is the main step in the proof of the classification theorem. The notation and terminology is that of $\S \S 4-6$.

7.2. REPARAMETRIZATION THEOREM. Suppose $(M, \phi),\left(M^{\prime}, \phi^{\prime}\right)$ are completely unstable $C^{r}(r \geqslant 1)$ dynamical systems, $h: M^{\prime} / \phi^{\prime} \rightarrow M / \phi$ is an order preserving homeomorphism, and $\left\{S_{n}\right\},\left\{S_{n}^{\prime}\right\}$ are compatible complete systems of cross-sections for $(M, \phi)$, $\left(M^{\prime}, \phi^{\prime}\right)$ respectively. Then there is a reparametrization $\tilde{\phi}$ of $\phi$, such that

$$
\tilde{f}_{i j}(\bar{x})=f_{i j}^{\prime}\left(h^{-1} \bar{x}\right) \quad\left(\text { all } i, j \in \mathbf{Z}^{+}, \bar{x} \in \pi\left(\tilde{S}_{i}\right) \cap \pi\left(\tilde{S}_{j}\right)\right),
$$

where $\tilde{f}_{i j}$ denotes the time from $\tilde{S}_{i}$ to $\tilde{S}_{j}$ relative to $\tilde{\phi}$, and $\left\{\tilde{S}_{n}\right\}$ is a shrinking of $\left\{S_{n}\right\}$.

7.3. EXAmple. Again the proof is lengthy, so we attempt to simplify the presentation by treating one of the main steps separately, namely the construction of a reparametrization of $\phi$ that correctly adjusts the time map for a single pair of cross-sections. We motivate some of the details of the construction with a simple 
example. Let $M=\left([-1,1] \times \mathbf{R}^{1}\right) \backslash\{\mathbf{a}, \mathbf{b}\}$, where $\mathbf{a}=(0,1), \mathbf{b}=(0,3)$. Let $\phi$ be the flow on $M$ generated by the differential equations $\dot{x}=0, \dot{y}=r(x, y)$, where $r$ : $[-1,1] \times \mathbf{R}^{1} \rightarrow[0,1]$ is a smooth function that vanishes exactly on $\{\mathbf{a}, \mathbf{b}\}$. Let $T, S$ be cross-sections of $\phi$ given by $T=[-1,1] \times\{0\}, S=[-1,1] \times\{4\}$, and let $f: T \backslash\{0\}$ $\rightarrow(0, \infty)$ denote the time from $T$ to $S$ relative to $\phi$ (see Figure 5). Set $\mathbf{x}=(x, 0) \in T$ for $x \in[-1,1]$. Given a continuous function $f^{\prime}: T \backslash\{0\} \rightarrow(0, \infty)$ such that $\lim _{x \rightarrow 0} f^{\prime}(\mathbf{x})=\infty$, we want to construct a reparametrization $\tilde{\phi}$ of $\phi$ that realizes $f^{\prime}$ as the adjusted time from $T$ to $S$. We define $\tilde{\phi}$ on $T \cdot \mathbf{R}^{1}=\{\phi(\mathbf{x}, t) \mid \mathbf{x}=(x, 0) \in T$, $\left.t \in \mathbf{R}^{1}\right\}$ by

$$
\tilde{\phi}_{t}=\rho^{-1} \tau_{t} \rho,
$$

where $\rho$ is a (fibrewise) homeomorphism of $T \cdot \mathbf{R}^{1}$ onto $T \times \mathbf{R}^{1}$ that maps each orbit segment $\mathbf{x} \cdot[0, f(\mathbf{x})]$ onto the vertical segment $\{\mathbf{x}\} \times\left[0^{\prime}, f(\mathbf{x})\right]$, and $\tau_{t}$ denotes the translation defined by $\tau_{t}(\mathbf{x}, s)=(\mathbf{x}, s+t)\left(\mathbf{x} \in T, s, t \in \mathbf{R}^{1}\right)$. We wish to extend $\tilde{\phi}$ to all of $M$ by requiring $\tilde{\phi}=\phi$ off $T \cdot \mathbf{R}^{1}$. Difficulties arise because $T \cdot \mathbf{R}^{1}$ is not closed in $M$. For example, while $\rho$ must map each orbit segment $\mathbf{x} \cdot[0, f(\mathbf{x})]$ onto $\{\mathbf{x}\} \times\left[0, f^{\prime}(\mathbf{x})\right]$, if $\rho^{-1} \tau_{t} \rho$ is to be extendable, then $\rho$ must map segments of the form $(x, 4) \cdot[-k, k]$ onto segments whose length more and more closely approximates $2 k$ as $x$ approaches 0 . Analogously, orbit segments which are close to a segment c $\cdot[\alpha, \beta](\mathbf{c}=(0,2)$, see Figure 5) must have a " $\tilde{\phi}$-length" close to $\beta-\alpha$ (i.e., close to their " $\phi$-length"). In the latter case we may insure this by placing an auxiliary cross-section $R$ through $\mathbf{c}$, and requiring that $\rho$ map segments of the form $\mathbf{r}$. $[-l(\mathbf{r}), l(\mathbf{r})]$ for $\mathbf{r}=(x, 2) \in R$ "isometrically" onto subintervals of $\{\mathbf{x}\} \times\left[0, f^{\prime}(\mathbf{x})\right]$ (where $\mathbf{r} \cdot[-l(\mathbf{r}), l(\mathbf{r})]$ is parametrized by $\phi$ and $\{\mathbf{x}\} \times\left[0, f^{\prime}(\mathbf{x})\right]$ is parametrized by the vertical coordinate). Here $l: R \rightarrow(0, \infty)$ must be chosen so that $\lim _{\mathbf{r} \rightarrow \mathbf{c}} l(\mathbf{r})=\infty$, but with $l(\mathbf{r})$ small enough that an isometric mapping of this segment of $\mathbf{x} \cdot[0, f(\mathbf{x})]$ into $\{\mathbf{x}\} \times\left[0, f^{\prime}(\mathbf{x})\right]$ is possible. If similar constraints are placed on the restrictions of $\rho$ to orbit segments through $\mathbf{s} \in S, \mathbf{t} \in T$, the flow $\phi$ defined by (*) on $T \cdot \mathbf{R}^{1}$ will have the desired extension to all of $M$.

The details of this informal description are carried out in the general case in the proof of Lemma 7.4.

7.4. Lemma (Adjustment of A SINGLE TIME MAP). Suppose $\left\{S_{n}\right\},\left\{S_{n}^{\prime}\right\}$ are compatible complete systems of cross-sections for $(M, \phi),\left(M^{\prime}, \phi^{\prime}\right)$, relative to the order preserving homeomorphism $h: M^{\prime} / \phi^{\prime} \rightarrow M / \phi$. Let $\left\{\tilde{S}_{n}\right\}$ be a shrinking of $\left\{S_{n}\right\}$. Then for fixed $n, j \in \mathbf{Z}^{+}$there is a reparametrization $\tilde{\phi}$ of $\phi$ such that the time $\tilde{f}_{n j}$ from $\tilde{S}_{n}$ to $\tilde{S}_{j}$ (with respect to $\left.\tilde{\phi}\right)$ satisfies

$$
\tilde{f}_{n j}(\bar{x})=f_{n j}^{\prime}\left(h^{-1}(\bar{x})\right) \quad\left(\bar{x} \in \pi\left(\tilde{S}_{n}\right) \cap \pi\left(\tilde{S}_{j}\right)\right) .
$$

Proof. We first establish our notation. For $x \in S_{n}$ let $\bar{x}$ denote $\pi(x) \in M / \phi$. Set $A=S_{j} \cdot \mathbf{R}^{1} \cap S_{n}, B=\tilde{S}_{j} \cdot \mathbf{R}^{1} \cap S_{n}$, and define mappings $f, f^{\prime}: A \rightarrow \mathbf{R}^{1}$ by

$$
f(x)=f_{n j}(\bar{x}), \quad f^{\prime}(x)=f_{n j}^{\prime}\left(h^{-1}(\bar{x})\right) .
$$

Let $A^{+}=\{x \in A \mid f(x)>0\}$ and $A^{-}=\{x \in A \mid f(x)<0\}$, so $A=A^{+} \cup A^{-} ; B=$ $B^{+} \cup B^{-}$analogously. 


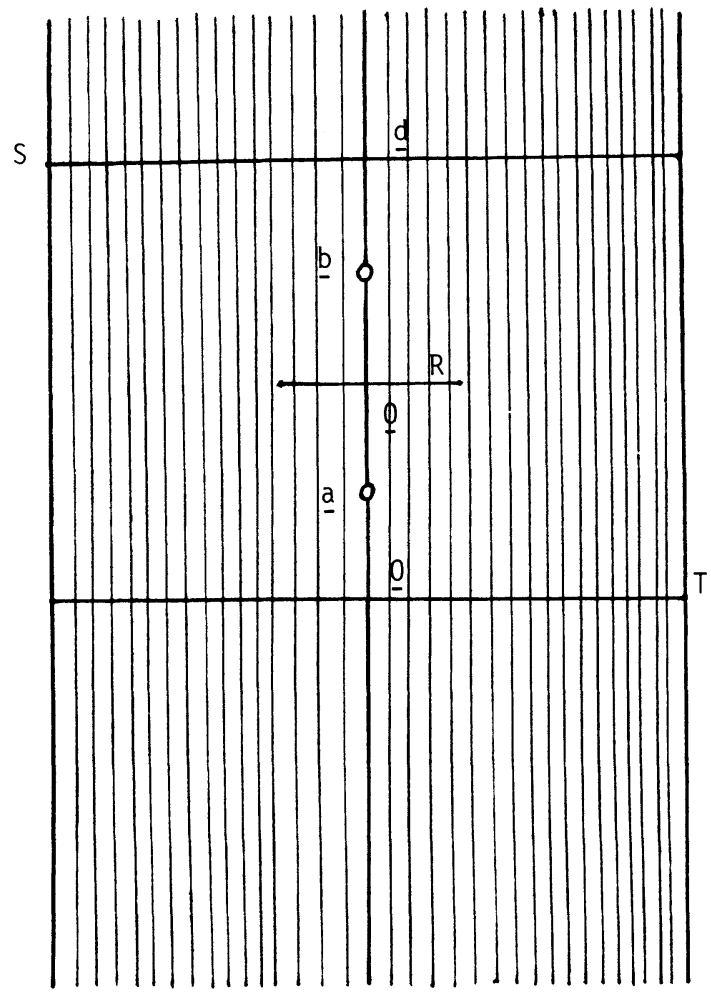

FIGURE 5

We will construct a homeomorphism $\rho: S_{n} \cdot \mathbf{R}^{1} \rightarrow S_{n} \times \mathbf{R}^{1}$ that maps each orbit $x \cdot \mathbf{R}^{1}$ (by an orientation preserving homeomorphism) onto the vertical fiber $\{x\} \times$ $\mathbf{R}^{1}$, taking $x \in S_{n}$ to $(x, 0) \in S_{n} \times \mathbf{R}^{1}$, taking $x \cdot[0, f(x)]$ onto $\{x\} \times\left[0, f^{\prime}(x)\right]$ if $x \in B^{+}$, and taking $x \cdot[f(x), 0]$ onto $\{x\} \times\left[f^{\prime}(x), 0\right]$ if $x \in B^{-}$. We then define $\tilde{\phi}_{t}$ for each $t \in \mathbf{R}^{1}$ by

$$
\tilde{\phi}_{t}(p)= \begin{cases}\rho^{-1} \tau_{t} \rho(p), & p \in S_{n} \cdot \mathbf{R}^{1}, \\ \phi_{t}(p), & p \in M \backslash S_{n} \cdot \mathbf{R}^{1},\end{cases}
$$

where $\tau_{t}: S_{n} \cdot \mathbf{R}^{1} \rightarrow S_{n} \times \mathbf{R}^{1}$ is (the vertical translation by $t$ ) defined by $\tau_{t}(x \cdot s)=$ $(x, t+s)\left(x \in S_{n} ; s, t \in \mathbf{R}^{1}\right)$. We will need to impose further restrictions on $\rho$ to insure that the mapping $\phi$ defined by $(*)$ is continuous at points of the boundary of $S_{n} \cdot \mathbf{R}^{1}$ in $M$, but if $\rho$ satisfies the preceding conditions, then it is easy to check that the restriction of $\phi$ to $S_{n} \cdot \mathbf{R}^{1}$ is a reparametrization of $\phi$ that satisfies the conclusion of 7.4.

We now proceed with the construction of $\rho$. We consider separately the construction on $S_{n} \cdot[0, \infty)$ and on $S_{n} \cdot(-\infty, 0]$, treating the case $S_{n} \cdot[0, \infty)$ in detail. Let $\bar{A}^{+}$ denote the closure of $A^{+}$in $S_{n}$, and let $K^{+}=\bar{A}^{+} \backslash A^{+}$. For each $m \geqslant n$ define $U_{m}$ by

$$
U_{m}=\left\{x \in A^{+} \mid f(x), f^{\prime}(x)>4(m+1)^{2}+2(m+1)\right\} ;
$$


$U_{m} \cup K^{+}$is a neighborhood of $K^{+}$in $\bar{A}^{+}$. For otherwise there is a sequence $\left\{x_{k}\right\}$ in $A^{+}$with $\left\{x_{k}\right\}$ converging to $x \in K^{+}$and $\left\{f\left(x_{k}\right)\right\}$ bounded. By taking subsequences we may assume there is a sequence $\left\{x_{k}\right\}$ in $A^{+}$, with $\left\{x_{k}\right\} \rightarrow x \in K^{+},\left\{f\left(x_{k}\right)\right\} \rightarrow t$ $\in[0, \infty)$ and (since $x_{k} \cdot f\left(x_{k}\right)$ is in the compact cross-section $S_{j}$ for each $k$ ) that $\left\{x_{k} \cdot f\left(x_{k}\right)\right\} \rightarrow x \cdot t \in S_{j}$. But then $f(x)=t$, and in fact $t>0$ (since $S_{j} \cap S_{n}=\varnothing$ ), so $x \in A^{+}$contrary to our initial assumption.

For each $m \geqslant n$ define $K_{m}=\left(S_{1} \cup \cdots \cup S_{m}\right) \cdot[-m, m]$; note that each $K_{m}$ is compact and $M=\bigcup_{m \geqslant n} K_{m}$. For each $x \in A^{+}$define

$$
c_{x}=\frac{1}{4} \min \left\{f(x), f^{\prime}(x)\right\}
$$

note that $c_{x}$ is a continuous function of $x \in A^{+}$. We now define $\rho$ "locally" as follows: for each $p \in \AA^{+}$choose $N_{p} \subseteq A^{+}$a closed $(m-1)$ disk that is a neighborhood of $p$ in $A^{+}$, and choose a homeomorphism $\rho_{p}: N_{p} \cdot[0, \infty) \rightarrow N_{p} \times[0, \infty)$, satisfying the following:

(a) If $N_{p} \cap U_{m} \neq \varnothing$, then $N_{p} \subseteq U_{m-1}$.

(b) For each $x \in N_{p}, \rho_{p}$ maps $x \cdot[0, \infty)$ onto $\{x\} \times[0, \infty)$.

(c) For each $x \in N_{p}, \rho_{p}$ maps $x \cdot\left[0, c_{x}\right]$ isometrically onto $\{x\} \times\left[0, c_{x}\right]$, and $x \cdot\left[f(x)-c_{x}, \infty\right)$ isometrically onto $\{x\} \times\left[f^{\prime}(x)-c_{x}, \infty\right)$ (here $x \cdot[0, \infty)$ is parametrized by $\phi$, and $\{x\} \times[0, \infty)$ is parametrized by the vertical coordinate).

(d) If $N_{p} \subseteq U_{m-1}$, then on each $x \cdot[0, \infty), \rho_{p}$ maps each component of $K_{m} \cap x$. $[0, f(x)]$ isometrically into $\{x\} \times\left[0, f^{\prime}(x)\right]$.

That such a choice is possible may be seen as follows.

We first choose the $N_{p}$ satisfying (a). If $N_{p} \subseteq U_{m-1}$ and $x \in N_{p}$, then the length of $x \cdot\left[c_{x}, f(x)-c_{x}\right]$ is at least $\frac{1}{2} f(x)>2 m^{2}+m ;\{x\} \times\left[c_{x}, f^{\prime}(x)-c_{x}\right]$ has length at least $\frac{1}{2} f^{\prime}(x)>2 m^{2}+m$. The number of components of $K_{m} \cap x \cdot\left[c_{x}, f(x)-c_{x}\right]$ is at most $m$, so their combined length cannot exceed $2 m^{2}$. Thus there is a homeomorphism of $x \cdot[0, f(x)]$ onto $\{x\} \times\left[0, f^{\prime}(x)\right]$ that satisfies the restrictions (b)-(d). This homeomorphism can be chosen to vary continuously as $x$ varies over $N_{p}$ because $f(x), f^{\prime}(x), c_{x}$, and the intersections $S_{i} \cap x \cdot[0, f(x)](i=1, \ldots, m)$ all vary continuously with $x$; for example the restriction of $\rho$ to each $x \cdot[0, \infty)$ can be taken to be a piecewise linear homeomorphism "canonically" constructed from this information. The details are straightforward but tedious, and hence we omit them.

Now let $\left\{\lambda_{\alpha}\right\}_{\alpha \in \mathscr{A}}$ be a locally finite partition of unity subordinate to the cover $\left\{\right.$ Int $\left.N_{p} \mid p \in \operatorname{Int} A^{+}\right\}$of Int $A^{+}$. For each $\alpha \in \mathscr{A}$ choose $p(\alpha)$ with $\operatorname{support}\left(\lambda_{\alpha}\right) \subseteq$ Int $N_{p(\alpha)}$ and define

$$
\rho_{1}=\sum_{\alpha \in \mathscr{A}} \lambda_{\alpha} \rho_{p(\alpha)}
$$

Then $\rho_{1}$ is a fibrewise homeomorphism of Int $A^{+} \cdot[0, \infty)$ onto Int $A^{+} \times[0, \infty)$ that satisfies the following for each $x \in \operatorname{Int} A^{+}$:

(e) $x \cdot\left[0, c_{x}\right]$ is mapped isometrically onto $\{x\} \times\left[0, c_{x}\right]$;

(f) $x \cdot\left[f(x)-c_{x}, \infty\right)$ is mapped isometrically onto $\{x\} \times\left[f^{\prime}(x)-c_{x}, \infty\right)$;

(g) If $x \in U_{m}$, then each component of $K_{m} \cap x \cdot[0, \infty)$ is mapped isometrically into $\{x\} \times[0, \infty)$. 
In fact each $\rho_{p}$ satisfies (e) and (f) (by (c) above), hence any convex combination does also. If $x \in U_{m}$ and $\lambda_{\alpha}(x) \neq 0$, then $\lambda_{\alpha}$ is supported on some $N_{p} \subseteq U_{m-1}$ (by (a) above), so $\rho_{p(\alpha)}$ satisfies (g) (by (d) above). Thus $\rho_{1}$ satisfies (g) also.

We now extend $\rho_{1}$ over all of $S_{n} \times[0, \infty)$ in several steps. We first extend to $A^{+} \cdot[0, \infty)$. Let $\delta A^{+}$denote $A^{+} \backslash$ Int $A^{+}$; note that $\delta A^{+}=A^{+} \cap \partial S_{j} \cdot \mathbf{R}^{1}$. Then $A^{+} \backslash B^{+}$ is a neighborhood in $A^{+}$of $\delta A^{+}$. Let $u_{1}: A^{+} \rightarrow[0,1]$ be a continuous (Urysohn) function satisfying $u_{1}(x)=0$ for $x \in \delta A^{+}$and $u_{1}(x)=1$ for $x \in B^{+}$. Let $\rho_{0}$ : $S_{n} \cdot[0, \infty) \rightarrow S_{n} \times[0, \infty)$ denote the homeomorphism defined by $\rho_{0}(x \cdot t)=(x, t)$. Define $\rho_{2}: A^{+} \cdot[0, \infty) \rightarrow A^{+} \times[0, \infty)$ by

$$
\rho_{2}=u_{1} \rho_{1}+\left(1-u_{1}\right) \rho_{0} .
$$

Then $\rho_{2}$ satisfies (f) above for $x \in B^{+}$, satisfies (e) and (g) for $x \in A^{+}$, and agrees with $\rho_{0}$ on $\delta A^{+} \cdot[0, \infty)$.

We can now extend $\rho_{2}$ to $\rho_{3}: \bar{A}^{+} \cdot[0, \infty) \rightarrow \bar{A}^{+} \times[0, \infty)$ by taking $\rho_{3}=\rho_{0}$ on $K^{+} \cdot[0, \infty)$; viz.,

$$
\rho_{3}(x \cdot t)= \begin{cases}\rho_{2}(x \cdot t), & x \in A^{+}, \\ \rho_{0}(x \cdot t), & x \in \bar{A}^{+} \backslash A^{+}=K^{+} .\end{cases}
$$

Again $\rho_{3}$ satisfies (f) on $B^{+}$, satisfies (e) and (g) on $\bar{A}^{+}$, and $\rho_{3}=\rho_{0}$ on $\left(\delta A^{+} \cup K^{+}\right) \cdot$ $[0, \infty)$

We may check that $\rho_{3}$ is continuous at $K^{+} \cdot[0, \infty)$ as follows. Suppose $\left\{x_{k}\right\}$ is a sequence in $A^{+}$that converges to $x \in K^{+}$, and $\left\{t_{n}\right\}$ is a sequence in $(0, \infty)$ that converges to $t \in[0, \infty)$. Choose $m \in \mathbf{Z}^{+}$so that $t_{1}, t_{2}, \ldots, t \in[0, m]$. Then there is a $k_{0} \in \mathbf{Z}^{+}$such that $x_{k} \in U_{m}$ for $k \geqslant k_{0}$, hence $t_{k} \in\left[0, c_{x_{k}}\right]$ for $k \geqslant k_{0}$. Since $\rho_{3}$ maps $x_{k} \cdot\left[0, c_{x_{k}}\right]\left(k \geqslant k_{0}\right)$ by an isometry it follows that $\rho_{3}\left(x_{k} \cdot t_{k}\right)=\left(x_{k}, t_{k}\right)$, which converges to $(x, t)=\rho_{3}(x \cdot t)$ as $k \rightarrow \infty$ as desired.

Note that the (topological) boundary $\bar{A}^{+} \backslash$ Int $A^{+}$of $A^{+}$in $S_{n}$ is just ( $A^{+} \cap \partial S_{j}$. $\left.\mathbf{R}^{1}\right) \cup K^{+}$. Thus $\rho_{3}$ agrees with $\rho_{0}$ on the boundary of $A^{+}$, and we can extend to $\rho_{4}$ : $S_{n} \cdot[0, \infty) \rightarrow S_{n} \times[0, \infty)$ by taking $\rho_{4}=\rho_{0}$ on all of $\left(S_{n} \backslash A^{+}\right) \cdot[0, \infty)$. We make one final adjustment to insure that $\rho=\rho_{0}$ on $\partial S_{n} \cdot[0, \infty)$. Let $u_{2}: S_{n} \rightarrow[0,1]$ be a continuous function satisfying $u_{2}(x)=0$ for $x \in \partial S_{n}$ and $u_{2}(x)=1$ for $x \in \tilde{S}_{n}$. Define $\rho$ by

$$
\rho=u_{2} \rho_{4}+\left(1-u_{2}\right) \rho_{0} .
$$

Then $\rho: S_{n} \cdot[0, \infty) \rightarrow S_{n} \times[0, \infty)$ is a fiberwise homeomorphism that satisfies (f) for $x \in \tilde{S}_{n} \cap B^{+}$, satisfies (e) and (g) for all $x \in S_{n}$ and agrees with $\rho_{0}$ over $\partial S_{n} \cup K^{+}$. We extend $\rho$ to $S_{n} \cdot(-\infty, 0]$ by an analogous construction.

We now define $\tilde{\phi}$ by $(*)$ and check that $\tilde{\phi}$ is the desired reparametrization of $\phi$. Because $\rho$ satisfies (f) (and the analogous condition when $f(x)$ is negative) for $x \in \tilde{S}_{n} \cap \tilde{S}_{j} \cdot \mathbf{R}^{1}$, we see that $\rho$ maps $x \cdot[0, f(x)]$ onto $\{x\} \times\left[0, f^{\prime}(x)\right]$ for $x \in \tilde{S}_{n} \cap$ $B^{+}$, and maps $x \cdot[f(x), 0]$ onto $\{x\} \times\left[f^{\prime}(x), 0\right]$ for $x \in \tilde{S}_{n} \cap B^{-}$. Thus

$$
\tilde{f}_{n j}(\bar{x})=f_{n j}^{\prime}\left(h^{-1} \bar{x}\right), \quad \bar{x} \in \pi\left(\tilde{S}_{n}\right) \cap \pi\left(\tilde{S}_{j}\right) .
$$

It remains to check the continuity of $\tilde{\phi}$ at points of the boundary of $S_{n} \cdot \mathbf{R}^{1}$ in $M$. At points of $\partial S_{n} \cdot \mathbf{R}^{1}$ there is no difficulty, since $\tilde{\phi}$ is clearly continuous on $S_{n} \cdot \mathbf{R}^{1}$, and $\tilde{\phi}=\phi$ on $\partial S_{n} \cdot \mathbf{R}^{1}$. Hence we consider a sequence $\left\{p_{k}\right\}$ in $S_{n} \cdot \mathbf{R}^{1}$ that converges to a 
point $p \notin S_{n} \cdot \mathbf{R}^{1}$, and a sequence $\left\{t_{k}\right\}$ of real numbers with $\left\{t_{k}\right\} \rightarrow t$. We show that $\left\{\tilde{\phi}\left(p_{k}, t_{k}\right)\right\} \rightarrow \tilde{\phi}(p, t)$. We may assume that $p_{k} \in S_{n} \cdot[0, \infty)$, say $p_{k}=\phi\left(x_{k}, s_{k}\right)$ with $x_{k} \in S_{n}$ and $s_{k}>0$. Since $p \notin S_{n} \cdot \mathbf{R}^{1}$ we must have that $\left\{s_{k}\right\} \rightarrow \infty$ (cf. the proof of Lemma 5.5). It follows that, for an arbitrary convergent subsequence $\left\{x_{j}\right\}$ of $\left\{x_{k}\right\}$, say $\left\{x_{j}\right\} \rightarrow x \in S_{n}$, we may assume $x \in K^{+}$. Now fix $i \in \mathbf{Z}^{+}$with $p \in$ Int $S_{i} \cdot \mathbf{R}^{1}$; then ultimately $p_{j} \in S_{i} \cdot \mathbf{R}^{1}$, and if $m$ is sufficiently large then there is a $j_{0} \in \mathbf{Z}^{+}$such that all the $p_{j}, \tilde{\phi}\left(p_{j}, t_{j}\right)$ for $j \geqslant j_{0}$, as well as $p, \tilde{\phi}(p, t)$ lie in $S_{i}[-m, m] \subseteq K_{m}$. Since $\left\{x_{j}\right\} \rightarrow x \in K^{+}$we may assume $j_{0}$ chosen so large that $x_{j} \in U_{m}$ for $j \geqslant j_{0}$. But then, as $\rho$ satisfies (g), we have that

$$
\tilde{\phi}\left(p_{j}, t_{j}\right)=\phi\left(p_{j}, t_{j}\right) \rightarrow \phi(p, t)=\tilde{\phi}(p, t) .
$$

As the convergent subsequence $\left\{x_{j}\right\}$ was arbitrary, it follows that $\tilde{\phi}\left(p_{k}, t_{k}\right) \rightarrow$ $\tilde{\phi}(p, t)$ for the full sequence, as required. This completes the proof of Lemma 7.4.

7.5. Notation. In the following proof of the reparametrization theorem we will use Lemma 7.4 repeatedly; each time we do we must give up a neighborhood of the boundary of certain cross-sections, so we first establish an infinite sequence of shrinkings. Let $\left\{\tilde{S}_{n}\right\}$ denote any fixed shrinking of $\left\{S_{n}\right\}$.

For each $n \in \mathbf{Z}^{+}$, choose a sequence $\left\{S_{n}^{k} \mid k \in \mathbf{Z}^{+}\right\}$of closed $(m-1)$ disks in Int $S_{n}$, with each $S_{n}^{k} \subseteq$ Int $S_{n}^{k-1}$, and $\cap\left\{S_{n}^{k} \mid k \in \mathbf{Z}^{+}\right\}=\tilde{S}_{n}$. Then for each fixed $k$, $\left\{S_{n}^{k} \mid \in \mathbf{Z}^{+}\right\}$is a shrinking of $\left\{S_{n}^{k-1} \mid n \in \mathbf{Z}^{+}\right\}$.

7.6. Proof of Reparametrization Theorem. We proceed by induction, with Lemma 7.4 providing the basis. To avoid excessive notation, each time we reparametrize we relabel so that the adjusted time maps are again denoted $\left\{f_{i j} \mid i, j \in \mathbf{Z}^{+}\right\}$. Note that $\left\{S_{n}\right\}$ remains a complete system compatible with $\left\{S_{n}^{\prime}\right\}$.

Thus assume that we have constructed reparametrizations $\phi_{1}, \ldots, \phi_{n-1}$ satisfying the following restrictions:

(a) For each $k \leqslant n-1$ we have $f_{i j}(\bar{x})=f_{i j}^{\prime}\left(h^{-1} \bar{x}\right)$ for all $i, j \leqslant k$ and all $\bar{x} \in \pi\left(S_{i}^{k}\right) \cap \pi\left(S_{j}^{k}\right)$;

(b) For each $k \leqslant n-1, \phi_{k}$ agrees with $\phi_{k-1}$ on the complement of $S_{k}^{k-1} \cdot \mathbf{R}^{1}$. Also, if $x \in S_{k}^{k-1}$ and $f_{k j}(x)>0$ for some $j<k$ and $x \in S_{j}^{k} \cdot \mathbf{R}^{1}$, then the time parametrization induced by $\phi_{k}$ on $y \cdot[0, \infty)$, where $y=\gamma(x) \cap S_{j}^{k}$, is the same as the time parametrization induced by $\phi_{k-1}$. Analogously for $x \in S_{k}^{k-1}$ with $f_{k j}(x)<0$ for some $j<k$.

(The latter restriction will be needed to prove that the sequence $\left\{\phi_{n}\right\}$ of reparametrizations that we construct does converge to a reparametrization of $\phi$.)

We now construct $\phi_{n}$ so that (a) and (b) are satisfied with $n$ in place of $n-1$. Here $\phi_{n}$ will be defined as in 7.4: we define a fiberwise homeomorphism $\rho: S_{n}^{n-1} \cdot \mathbf{R}^{1} \rightarrow$ $S_{n}^{n-1} \times \mathbf{R}^{1}$ and set

$$
\left(\phi_{n}\right)_{t}(p)= \begin{cases}\rho^{-1} \tau_{t} \rho(p), & p \in S_{n}^{n-1} \cdot \mathbf{R}^{1}, \\ \phi_{n-1}(p), & p \in M \backslash S_{n}^{n-1} \cdot \mathbf{R}^{1} .\end{cases}
$$

We treat the construction of $\rho$ on $S_{n}^{n-1} \cdot[0, \infty)$ in detail, the construction on $S_{n}^{n-1} \cdot(-\infty, 0]$ being analogous. Set $T=S_{n}^{n-1}$, and let $\theta: T \cdot \mathbf{R}^{1} \rightarrow T$ denote the natural projection. For each $j \leqslant n$ define open sets $O_{j}^{\prime}, Q_{j}^{\prime}, R_{j}^{\prime} \subseteq S_{j}$ so that

$$
S_{j}^{n}=P_{j}^{\prime} \subseteq O_{j}^{\prime} \subseteq \bar{O}_{j}^{\prime} \subseteq Q_{j}^{\prime} \subseteq \bar{Q}_{j}^{\prime} \subseteq R_{j}^{\prime}=\text { Int } S_{j}^{n-1}
$$


(where the closure and interior are taken with respect to $S_{j}$ ). For each $j$ set $A_{j}^{+}=\left\{x \in T \mid f_{n j}(x)>0\right\}$, and define (cf. Figure 6):

$$
\begin{array}{ll}
P_{j}=\theta\left(P_{j}^{\prime}\right) \cap A_{j}^{+}, & O_{j}=\theta\left(O_{j}^{\prime}\right) \cap A_{j}^{+}, \\
Q_{j}=\theta\left(Q_{j}^{\prime}\right) \cap A_{j}^{+}, & R_{j}=\theta\left(R_{j}^{\prime}\right) \cap A_{j}^{+} .
\end{array}
$$

Note that $O_{j}, Q_{j}, R_{j}$ are all open in $T$.

Let $\rho_{0}: T \cdot[0, \infty) \rightarrow T \times[0, \infty)$ denote the homeomorphism defined by $\rho_{0}(x \cdot t)$ $=(x, t)$. For $j=1,2, \ldots, n-1$ we use Lemma 7.4 (and its proof) to obtain fiberwise homeomorphisms $\rho_{j}$ of $T \cdot[0, \infty)$ onto $T \times[0, \infty)$ satisfying the following:

(a1) $\rho_{j}$ adjusts $f_{n j}$ to $f_{n j}^{\prime}$ over $O_{j} \cap S_{n}^{n}$;

(b1) $\rho_{j}=\rho_{0}$ over $T \backslash Q_{j}$;

(c1) $\rho_{j}=\rho_{0}$ over a neighborhood of $\partial T$;

(d1) $\rho_{j}$ maps $x \cdot\left[f_{n j}(\bar{x}), \infty\right)$ by an isometry for each $x \in A_{j}^{+}$; and

(e1) the reparametrization defined by $\rho_{j}^{-1} \tau_{t} \rho_{j}(t \geqslant 0)$ on $T \cdot[0, \infty)$ extends continuously by $\left(\phi_{n-1}\right)_{t}$ on $(M \backslash T) \cdot[0, \infty)$.

Define $\rho_{n}=\rho_{0}$. Since we are constructing a fiberwise homeomorphism $\rho$ that simultaneously adjusts $(n-1)$ maps $f_{n i}(1 \leqslant i \leqslant n-1)$, we may assume by induction that we can construct such homeomorphisms that simultaneously adjust any $(n-2)$ of the $f_{n i}$. Thus suppose that we have fiberwise homeomorphisms $\rho_{n+j}$ : $T \cdot[0, \infty) \rightarrow T \times[0, \infty)$ for $j=1, \ldots, n-1$, satisfying the following:

(a2) $\rho_{n+j}$ adjusts each $f_{n i}(i \neq j, i \leqslant n-1)$ to $f_{n i}^{\prime}$ on $P_{i} \cap S_{n}^{n}$;

(b2) $\rho_{n+j}=\rho_{0}$ over $T \backslash \bigcup_{i \neq j} Q_{i}$;

(c2) $\rho_{n+j}=\rho_{0}$ over a neighborhood of $\partial T$;

(d2) for each $i \neq j, i \leqslant n-1, \rho_{n+j}$ maps $x \cdot\left[f_{n i}(\bar{x}), \infty\right)$ by an isometry, for each $x \in P_{i}$; and

(e2) the reparametrization defined by $\rho_{n+j}^{-1} \tau_{t} \rho_{n+j}(t \geqslant 0)$ on $T \cdot[0, \infty)$ extends continuously by $\left(\phi_{n-1}\right)_{t}$ on $(M \backslash T) \cdot[0, \infty)$.

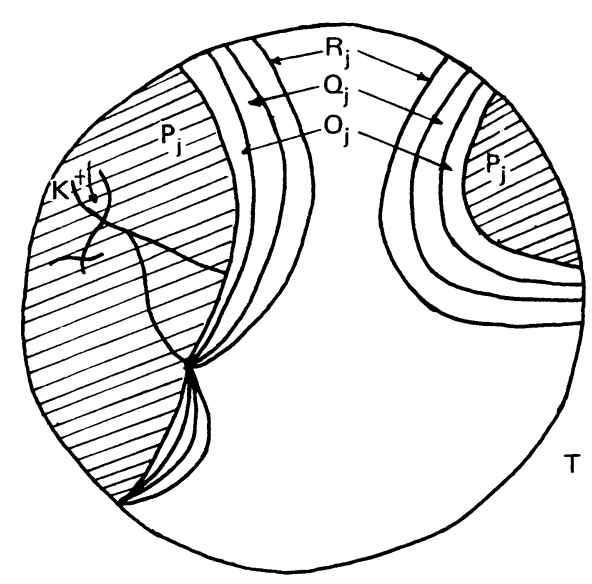

FigURE 6 
We obtain the desired homeomorphism by piecing together $\rho_{1}, \ldots, \rho_{2 n-1}$ by means of a partition of unity.

Define $Q=\bigcup_{j \leqslant n-1} Q_{j}$, and define $u: Q \rightarrow \mathbf{R}^{1}$ by $u(x)=\min \left\{f_{n j}(x) \mid f_{n j}(x)>0\right.$, $j \leqslant n-1\}$. Set

$$
K^{+}=\left\{x \in T \mid \text { for some sequence }\left\{x_{k}\right\} \text { in } Q,\left\{x_{k}\right\} \rightarrow x,\left\{u\left(x_{k}\right)\right\} \rightarrow \infty\right\} .
$$

Then $K^{+}$is closed in $T$, and has the following property: If $x \in K^{+}$and $\beta>0$, then there is a neighborhood $N$ of $x$ in $T$, such that $f_{n j}(y)>\beta$ for all $y \in N \cap P_{j}$. We first define $\rho$ over $T \backslash K^{+}$. For each $j=1, \ldots, n-1$ define

$$
\begin{gathered}
V_{j}=\left\{x \in O_{j} \mid \text { if } f_{n i}(x)<f_{n j} \text { for some } i \leqslant n-1, \text { then } x \notin P_{i}\right\}, \\
V_{n+j}=\left(R_{j} \backslash P_{j}\right) \cap\left(T \backslash K^{+}\right),
\end{gathered}
$$

and for $j=n$ define

$$
V_{n}=\left(T \backslash K^{+}\right) \backslash\left(\bar{P}_{1} \cup \cdots \cup \bar{P}_{n-1}\right)
$$

(where the bar denotes the closure in $\left.T \backslash K^{+}\right)$. Then each $V_{j}(j=1, \ldots, 2 n-1)$ is open in $T \backslash K^{+}$, and in fact $\left\{V_{j} \mid j \leqslant 2 n-1\right\}$ covers $T \backslash K^{+}$. To see this fix $x \in T \backslash K^{+}$. If $x$ is in some $P_{i}$, choose $j$ so $f_{n j}(x)=\min \left\{f_{n i}(x) \mid x \in P_{i}\right\}$; then $x \in V_{j}$. Thus we may assume $x$ is in no $P_{i}$. Also we may as well assume $x \notin V_{n}$. But then $x \in \bar{P}_{j}$ for some $j \leqslant n-1$, so $x \in \bar{P}_{j} \backslash P_{j}$, and there is a sequence $\left\{x_{k}\right\}$ in $Q$ with $\left\{x_{k}\right\} \rightarrow x$, and $f_{n j}\left(x_{k}\right) \rightarrow \infty$. Since $x \notin K^{+}$this implies that $x \in R_{i}$ for some $i \neq j$. But then $x \in V_{n+i}$.

Now let $\left\{\lambda_{j} \mid j \leqslant 2 n-1\right\}$ be a partition of unity subordinate to this open cover of $T \backslash K^{+}$, and define

$$
\rho=\sum_{j \leqslant 2 n-1} \lambda_{j} \rho_{j}
$$

We verify that $\rho$ has all the desired properties.

(i) For $j \leqslant n-1, \rho$ adjusts $f_{n j}$ to $f_{n j}^{\prime}$ on $P_{j} \cap S_{n}^{n}$. Fix $x \in P_{j} \cap S_{n}^{n}$. If $\lambda_{i}(x) \neq 0$ for $i \leqslant n-1$, then $x \in V_{i}$. Since also $x \in P_{j}$ we must have $f_{n j}(x) \geqslant f_{n i}(x)$ (with equality only if $i=j$ ), and therefore $\rho_{i}$ does adjust $f_{n j}$ to $f_{n j}^{\prime}$ (by (a1) it maps $x \cdot f_{n i}(\bar{x})$ to $\left(x, f_{n i}^{\prime}\left(h^{-1} \bar{x}\right)\right)$, and maps $x \cdot\left[f_{n i}(\bar{x}), \infty\right)$ by an isometry, while $f_{i j}(\bar{x})=$ $f_{i j}^{\prime}\left(h^{-1} \bar{x}\right)$ by induction). Since $x \in P_{j}$ we must have $\lambda_{n}(x)=0$ (support $\left(\lambda_{n}\right) \cap P_{j}=$ $\varnothing)$. Finally suppose $\lambda_{n+i}(x) \neq 0$ for $i \leqslant n-1$. Then $x \in V_{n+i}$. Again $i \neq j$ since $V_{n+j} \cap P_{j}=\varnothing$. But then by (a2) $\rho_{n+i}$ does adjust $f_{n j}$ to $f_{n j}^{\prime}$ on the orbit of $x$. Thus $\rho$ is a convex combination of homeomorphisms each adjusting $f_{n j}$ to $f_{n j}^{\prime}$ over $\{x\}$.

(ii) The extension of $\rho$ to $T \cdot[0, \infty)$ by $\rho_{0}$ over $K^{+}$is a homeomorphism. For if $x \in K^{+}$, then $x$ is in no $Q_{i}$, so (by (b1) and (b2)) all of the $\rho_{j}$ agree with $\rho_{0}$ on the orbit of $x$. Assertion (ii) now follows from the continuity of the $\rho_{j}$.

For the remainder of the proof $\rho$ denotes the extended homeomorphism.

(iii) Over points of $T \backslash \bigcup_{i<n-1} R_{i}$ and on a neighborhood of $\partial T, \rho$ agrees with $\rho_{0}$. This follows from (c1), (c2), and the fact that, for $i \leqslant n-1 \operatorname{support}\left(\lambda_{j}\right) \subseteq O_{i} \subseteq R_{i}$, $\operatorname{support}\left(\lambda_{n+i}\right) \subseteq R_{i}$.

(iv) For $j \leqslant n-1$, if $x \in P_{j}$ then $\rho$ maps $x \cdot\left[f_{n j}(\bar{x}), \infty\right)$ by an isometry. The argument here is essentially as in (i) above using (d1) and (d2). 
(v) The reparametrization defined by $\rho^{-1} \tau_{t} \rho(t \geqslant 0)$ on $T \cdot[0, \infty)$ extends continuously by $\left(\phi_{n-1}\right)_{t}$ on $(M \backslash T) \cdot[0, \infty)$. This follows immediately from (e1) and (e2).

Note first that (i)-(v) justify our use of induction to obtain $\rho_{n+1}, \ldots, \rho_{2 n-1}$ above. We now assume that $\rho$ has been constructed analogously to $T \cdot(-\infty, 0]$, and define $\phi_{n}$ by

$$
\left(\phi_{n}\right)_{t}(p)= \begin{cases}\rho^{-1} \tau_{t} \rho(p), & p \in T \cdot \mathbf{R}^{1}, \\ \phi_{t}(p), & p \in(M \backslash T) \cdot \mathbf{R}^{1} .\end{cases}
$$

It follows from (i) that (a) is satisfied. It follows from (iii)-(v) that (b) is satisfied. Thus our induction is complete, and $\phi_{n}$ is the desire reparametrization.

Finally we must show that the sequence $\left\{\phi_{n}\right\}$ of reparametrizations determined by this construction in fact converges to a reparametrization of $\phi$ that adjust all the time maps simultaneously, at least over the shrinking $\left\{\tilde{S}_{n}\right\}$ of $\left\{S_{n}\right\}$. In fact we will verify that $\left\{\phi_{n}\right\}$ is locally finite in the sense that for any $(x, t) \in M \times \mathbf{R}^{1}$ there are neighborhoods $N \subseteq M$ of $x, I \subseteq \mathbf{R}^{1}$ of $t$, and an index $n_{0}$ such that $\phi_{n}=\phi_{n_{0}}$ for all $(y, s) \in N \times I$, and all $n \geqslant n_{0}$. Thus the pointwise limit of $\left\{\phi_{n}\right\}$ is a well-defined reparametrization of $\phi$; it is easy to see that it correctly adjusts all the time maps.

To see that $\left\{\phi_{n}\right\}$ is locally finite, fix $x \in M, t \in \mathbf{R}^{1}$, for definiteness say $t>0$. Because $\left\{\tilde{S}_{n}\right\}$ is complete we may choose cross-sections $S_{i}, S_{j}$ so that there is a compact neighborhood $N \subseteq \operatorname{Int} \tilde{S}_{i} \cap\left(\right.$ Int $\left.\tilde{S}_{j} \cdot \mathbf{R}^{1}\right)$ of $\gamma(x) \cap S_{i}$, with $f_{i j}$ positive on $N$, and both $x, x \cdot t$ in the flow box:

$$
F=\left\{y \cdot s \mid y \in N, s \in\left[0, f_{i j}(y)\right]\right\}
$$

(here $f_{i j}$ and $y \cdot s$ may refer to the original $\phi$; but not that $F$ is well defined independent of any reparametrization of $\phi$ ). Since $\left\{S_{n}\right\}$ is locally finite only finitely many of the $S_{n}$ meet $F$. Let $n_{0}$ be the largest index of those that do. Now since $\left\{\phi_{n}\right\}$ satisfies (b), if $n>n_{0}$ then the time parametrizations of an orbit segment in $F$ induced by $\phi_{n}$ and $\phi_{n-1}$ are identical. Thus if $(y, s)$ is sufficiently close to $(x, t)$, then $\phi_{n}(y, s)=\phi_{n_{0}}(y, s)$ for all $n>n_{0}$ as required. This completes the proof of the reparametrization theorem.

8. An application. We now give an application of the classification theorem proved above. In [Wi] Wilson gives a necessary and sufficient condition for local equivalence of flows and uses it to prove that Coleman's conjecture (stated below) holds if either $m=1$ or $n=1$. We give a simple proof of this fact using the classification theorem.

8.1. The conjecture of Coleman. A flow $\phi$ on $\mathbf{R}^{m} \times \mathbf{R}^{n}$ is said to have 0 as a topologically hyperbolic equilibrium if

(a) $\{0\}$ is the maximal $\phi$-invariant subset with isolating block $B=D^{m} \times D^{n}$.

(b) The set of points at which the vector field is (externally) tangent to $B$ is

$$
\tau=\partial D^{m} \times \partial D^{n}=\left\{(x, y) \in \mathbf{R}^{m+n} \mid\|x\|=1,\|y\|=1\right\} .
$$

(c) Orbits of $\phi$ enter $B$ on $b^{+} \backslash \tau$ (the set of ingress points), where $b^{+}=\partial D^{m} \times D^{n}$, and leaves $B$ on $b^{-} \backslash \tau$ (the set of egress points), where $b^{-}=D^{m} \times \partial D^{n}$. 

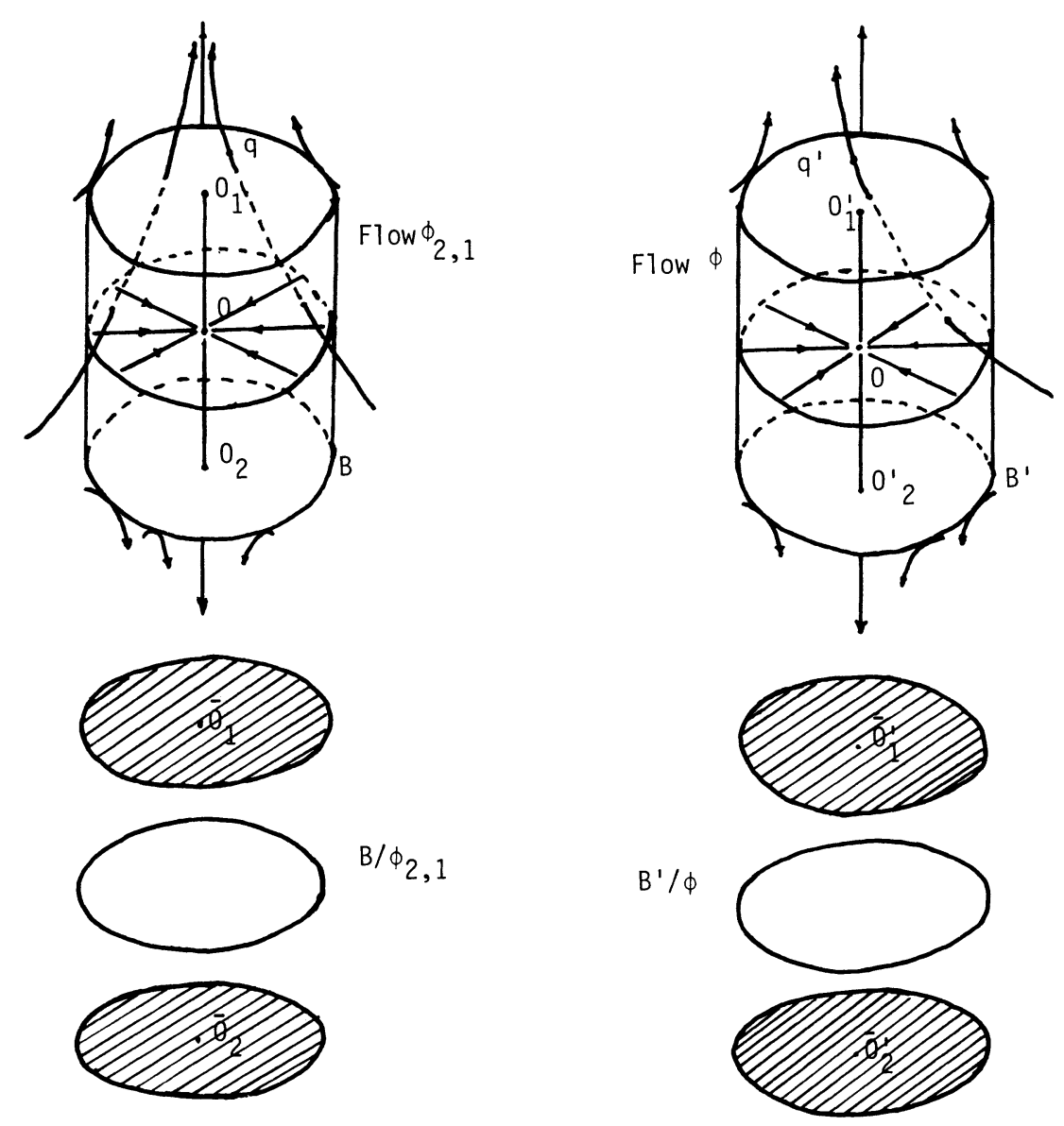

FIGURE 7

(d) The stable (unstable) manifold of 0 in $B$ is $D^{m} \times\{0\}\left(\{0\} \times D^{n}\right)$ that is for $x \in B$, the orbit $\gamma(x)$ tends to 0 in positive (negative) time if and only if $x \in D^{m} \times$ $\{0\}\left(x \in\{0\} \times D^{n}\right)$.

CONJECTURE. Such a flow $\phi$ is locally topologically equivalent at 0 to the standard hyperbolic flow $\phi_{m, n}$ on $\mathbf{R}^{m} \times \mathbf{R}^{n}$ defined by the differential equations

$$
\dot{x}=-x, \quad \dot{y}=y \quad\left(x \in \mathbf{R}^{m}, y \in \mathbf{R}^{n}\right),
$$

where $\phi$ is said to be locally topologically equivalent (at 0$)$ to $\phi_{m, n}$ if there is a homeomorphism $h: U \rightarrow h(U)$ of some neighborhood $U$ of 0 that takes each orbit segment of $\phi$ in $U$ onto an orbit segment of $\phi_{m, n}$ in $h(U)$ preserving the natural orientation.

8.2. REMARK. In [Ne3], Neumann gives a counterexample to the conjecture by constructing a $C^{\infty}$ flow $\phi$ on $\mathbf{R}^{2} \times \mathbf{R}^{2}$ that is not locally equivalent to the correspondiing standard hyperbolic flow $\phi_{2,2}$.

The conjecture is now fully resolved and there exist counterexamples in all higher dimensions [Pi]. 
8.3. Proof of The Conjecture When $n=1$. We want to show that $\phi_{m, 1}$ is locally topologically equivalent at 0 to $\phi$. Let $B \cong D^{m} \times D^{1}$ and $B^{\prime} \cong D^{m} \times D^{1}$ be the given isolating blocks at 0 for $\left(\mathbf{R}^{m+1}, \phi_{m, 1}\right)$ and $\left(\mathbf{R}^{m+1}, \phi\right)$ respectively. We will find a local equivalence of $B$ onto $B^{\prime}$. Since any local equivalence $k: B /\{0\} \rightarrow B^{\prime} /\{0\}$ automatically extends by $k(0)=0$, we can consider the completely unstable restrictions of $\phi_{m, 1}$ and $\phi$ to $\mathbf{R}^{m+1} /\{0\}$. By the classification theorem it then suffices to find an order preserving homeomorphism of the orbit spaces

$$
h: N / \phi_{m, 1} \rightarrow N^{\prime} / \phi,
$$

where $N=B /\{0\}$ and $N^{\prime}=B^{\prime} /\{0\}$.

Since 0 is topologically hyperbolic for both $\phi_{m, 1}$ and $\phi$, both orbit spaces may be regarded as quotient spaces of (cf. Figure 7)

$$
\partial\left(D^{m} \times D^{1}\right)=\left(S^{m-1} \times D^{1}\right) \cup\left(D^{m} \times S^{0}\right)
$$

in which $S^{m-1} \times\left(D^{1} \backslash\{0\}\right)$ is identified with $\left(D^{m} \backslash\{0\}\right) \times S^{0}$ by means of the Poincaré map induced by $\phi_{m, 1}$ in the case of $N / \phi_{m, 1}$ and by $\phi$ in the case of $N^{\prime} / \phi$. We will denote these Poincaré maps by $p, p^{\prime}$ respectively; thus, for $x \in S^{m-1} \times D^{1}$, the $\phi_{m, 1}$ orbit that enters $N$ at $x$ exists $N$ at $p(x)$,

$$
N / \phi_{m, 1} \cong\left(S^{m-1} \times D^{1}\right) \cup\left(D^{m} \times S^{0}\right) /\{x \leftrightarrow p(x)\},
$$

and

$$
N^{\prime} / \phi \cong\left(S^{m-1} \times D^{1}\right) \cup\left(D^{m} \times S^{0}\right) /\left\{x \leftrightarrow p^{\prime}(x)\right\} .
$$

We now obtain the homeomorphism $h: N / \phi_{m, 1} \rightarrow N^{\prime} / \phi$ from a homeomorphism $h_{1}$ of $\left(S^{m-1} \times D^{1}\right) \cup\left(D^{m} \times S^{0}\right)$ onto itself that is compatible with these identifications. On $S^{m-1} \times D^{1}$ we can take $h_{1}$ to be the "identity"; because of the compatibility requirement this automatically induces

$$
h_{1}:\left(D^{m} \backslash\{0\}\right) \times S^{0} \rightarrow\left(D^{m} \backslash\{0\}\right) \times S^{0}
$$

by $h_{1}=p^{\prime} h_{1} p^{-1}$. Now observe that this automatically extends to the required homeomorphism of all of $\partial M$ onto $\partial M^{\prime}$. The induced homeomorphism $h: N / \phi_{m, 1} \rightarrow$ $N^{\prime} / \phi$ clearly preserves the order structure, and so the proof is complete.

\section{BIBLIOGRAPHY}

[AD] A. Antosiewicz and J. Dugundgi, Parallelizable flows and Liapunov's second method, Ann. of Math. (2) 73 (1961), 543-555.

[Bi] R. H. Bing, The Cartesian product of a certain non-manifold and a line in $E^{4}$, Ann. of Math. (2) 70 (1959), 399-412.

[BS] N. P. Bhatia and G. P. Szegö, Stability theory of dynamical systems, Springer-Verlag, New York, 1970.

[Ch] W. C. Chewning, A dynamical system on $E^{4}$ neither isomorphic nor equivalent to a differentiable system, Bull. Amer. Math. Soc. 80 (1974), 150-154.

[C0] W. C. Chewning and R. Steven Owen, Local sections of flows on manifolds, Proc. Amer. Math. Soc. 49 (1975), 71-77.

[Du] J. Dugundgi, Topology, Allyn and Bacon, Boston, Mass., 1967.

[Go] Sudhir K. Goel, Complete unstable flows on manifolds, Ph.D. dissertation.

[HR] A Haefliger and G. Reeb, Variétés (non séparaées) à une dimension et structures feuilletées du plan, Enseign. Math. (2) 3 (1957), 107-125.

[Ma] L. Markus, Parallel dynamical systems, Topology 8 (1969), 47-57. 
[Ne1] Dean A. Neumann, Completely unstable flows on two manifolds, Trans. Amer. Math. Soc. 225 (1977), 211-226.

[Ne2] __ Smoothing continuous flows, J. Differential Equations 24 (1977), 127-135.

[Ne3] _ Topologically hyperbolic equilibria in dynamical systems, J. Differential Equations 37 (1980), 49-59.

[Ni1] Z. Nitecki, Explosions in completely unstable dynamical systems, Proc. Univ. of Florida, Internat. Sympos. A. R. Cesari, ed., Academic Press, New York, 1977, pp. 473-475.

[Ni2] _ Explosions in completely unstable flows. I. Preventing explosions, Trans. Amer. Math. Soc. 245 (1978), 43-61.

[Ni3] $\ldots$ Explosions in completely unstable flows. II. Some examples, Trans. Amer. Math. Soc. 245 (1978), 63-88.

[Ni4]__ Bifurcation from completely unstable flows on the cylinder. Bifurcation theory and applications in scientific disciplines, Ann. New York Acad. Sci. 316 (1979), 86-107.

[Pi] D. Pixton, More counterexamples to the Coleman conjecture, Proc. Amer. Math. Soc. 82 (1981), $145-148$.

[St] N. Steenrod, The topology of fiber bundles, Princeton Univ. Press, Princeton, N. J., 1951.

[TW] F. Takens and W. White, Vector fields with no nonwandering points, Amer. J. Math. 98 (1976), 415-425.

[Wa] T. Wazewski, Sur un problème de caractère intégral relatif à l'équation $\partial z / \partial x+Q(x, y) \partial z / \partial y=0$, Mathematica 8 (1934), 103-116.

[Wh1] H. Whitney, Regular families of curves, Ann. of Math. 34 (1933), 244-270.

[Wh2] _ Cross-sections of curves in 3-space, Duke Math. J. 4 (1938), 222-226.

[Wi] F. W. Wilson, A uniform continuity condition which is equivalent to Coleman's conjecture, J. Differential Equations 36 (1980), 12-19.

Department of Applied Mathematical Sciences, University of Houston-Downtown, HousTON, TEXAS 77002

Department of Mathematics, Bowling Green State University, Bowling Green, Ohio 43403 